\title{
Accurate recovery-based upper error bounds for the extended finite element framework
}

\author{
J.J. Ródenas ${ }^{\text {a,* }}$, O.A. González-Estrada ${ }^{\text {a }}$, P. Díez ${ }^{\text {, }}$ F.J. Fuenmayor ${ }^{\text {a }}$
}

\section{A B S T R A C T}

This paper introduces a recovery type error estimator yielding upper bounds of the error in energy norm for linear elastic fracture mechanics problems solved using the extended finite element method (XFEM). The paper can be considered as an extension and enhancement of a previous work in which the upper bounds of the error were developed in a FEM framework. The upper bound property requires the recovered solution to be equilibrated and continuous. The proposed technique consists of using a recovery technique, especially adapted to the XFEM framework that yields equilibrium at a local level (patch by patch). Then a postprocess based on the partition of unity concept is used to obtain continuity. The result is a very accurate but only nearly statically admissible recovered stress field, with small equilibrium defaults introduced by the postprocess. Sharp upper bounds are obtained using a new methodology accounting for the equilibrium defaults, as demonstrated by the numerical tests.

Keywords:

Extended finite element method A posteriori error estimation Linear elastic fracture mechanics Equilibrated stresses, Upper error bol

\section{Introduction}

The eXtended Finite Element Method (XFEM) is currently a well established very efficient numerical technique for modelling inclu sions and cracks $[1,39]$. With respect to the standard Finite Element Method (FEM), XFEM has the advantage of making the finite element mesh independent of the crack geometry. Thus, the mesh does not need to be modified during the crack propagation process. The XFEM uses the partition of unity concept [2] to add new interpolation functions that enrich the mathematical representation of the solution in the vicinity of the crack.

The first developments in this method [1,39] were soon followed by a considerable amount of publications as described in [3]. Thanks to all these developments and to the latter contributions in this field (see for example [4 11]) the XFEM is now considered to be a robust and highly accurate tool to analyze Linear Elastic Fracture Mechanics (LEFM) problems.

As in the FEM, the results obtained using the XFEM are affected by the discretization error associated with the finite size of the elements. In fact, this technique tends to be used with coarse discretizations. Thus, it is necessary to quantitatively assess their quality and, consequently, error estimation techniques are also required in the XFEM context.

\footnotetext{
* Corresponding author. Centro de Investigación de Tecnología de Vehículos. Departamento de Ingeniería Mecánica y de Materiales.Universidad Politécnica de Valencia, 46022-Valencia, Spain. Tel.: +34 963877000x86211; fax: +34 963877629. E-mail address: jjrodena@mcm.upv.es (J.J. Ródenas).
}

\subsection{Error assessment in the FEM}

The error assessment tools for Finite Element analysis are already standard. These tools are classified $[12,13]$ mainly into two families: residual type error estimators and recovery based error estimators.

Although the $a$ posteriori implicit residual type estimators have a sounder mathematical basis, the recovery based estimates, based on the ideas of Zienkiewicz and Zhu[14] and, in particular, those based on the Superconvergent Patch Recovery technique (SPR) $[15,16]$, are often preferred by practitioners due to their simple implementation and robustness $[17,18]$.

Since the publication of the SPR technique [15], numerous authors [19 21] have proposed new recovery techniques based on the results obtained from the FE analyses. Xiao and Karihaloo[22] presented an extensive literature review of this kind of techniques. Refs. [23] and [24] are especially relevant for the developments presented in this paper.

Ref. [23] presents the SPR C recovery technique. It consists in enforcing internal and boundary equilibrium equations and the com patibility equation at each patch. These additional constraints in the stress patch recovery are implemented using the Lagrange multipliers technique.

Ref. [24] describes the SPR CK technique which is an adaptation of the SPR C technique to linear elasticity problems with singularities solved with FEM. This adaptation is based on two fundamental aspects:

a) The stress field is decomposed into a singular field and a smooth field (smooth + singular stress field splitting) around the crack tip. 
Then, a different recovery technique is used for each component: the SPR C technique for the smooth part, and a reconstruction of the singular field using the stress intensity factors (SIFs) $K_{\mathrm{I}}$ and $K_{\mathrm{II}}$ computed from the interaction integral $[25,26]$ for the singular part.

b) Use of the conjoint polynomial enhancement proposed by Blacker and Belytschko [21]. This is a PUM based technique that ade quately weights the different values of recovered stresses obtained at each point from different patches.

\subsection{Upper error bounds in the FEM}

The residual type error estimators build up a statically admissible stress field and furnish upper bounds of the energy norm of the error $[12,13,27,28]$. This has been a traditional advantage of the residual type estimators over recovery based error estimators which were unable to produce guaranteed upper bounds of the error in energy norm.

Other approach aims at obtaining upper error bounds involving dual analysis that directly provides an equilibrated solution. This solution is compared with the standard and compatible finite element solution in order to assess the error $[29,30]$. The obtained estimate is therefore an upper error bound.

Ref. [31] introduces a recovery based technique that provides practical upper error bounds for the FEM framework. The technique is based on obtaining a recovered equilibrated stress field. The technique proposed in this reference provides a very accurate estimation of the error in energy norm and, additionally, a practical upper bound of the error that shows a good behaviour in problems with smooth solutions. Nevertheless, if this approach is used in problems containing singularities, the effectivity of the estimate is not guaranteed in a mesh refinement procedure.

\subsection{Error assessment tools in the XFEM}

The literature on error estimation methods for mesh based PUMs, however, is very limited. Xiao et al. [10] propose an approach based on the use of the Moving Least Squares (MLS) technique adapted to the XFEM framework and the use of statically admissible basis functions to obtain a recovered stress field that improves the accuracy of the stresses obtained by XFEM. In any case, these authors do not use the recovered stress field to obtain an error estimate.

Strouboulis et al. [9] propose the use of a recovery type error estimator for the Generalized Finite Element Method (GFEM) yielding good results for $h$ adapted meshes. A later proposal includes two new a posteriori error estimators for GFEM [32]. The first one is based on patch residual indicators and provides an accurate theoretical upper bound estimate, but its computed version severely underestimates the exact error. The second one is an error estimator based on a recovered displacement field and its performance is closely related to the quality of the GFEM solution.

Bordas and co workers [33 35 ] have recently developed recovery techniques adapted to the XFEM framework yielding very accurate estimations of the error in energy norm but lacking from the equilib rium constraints needed to guarantee the upper bound property. The first technique is a MLS approach that introduces a visibility criterion and a MLS basis enriched with crack tip functions, and the second one is a global derivative recovery formulation extended to XFEM problems.

Recently, a technique that provides local error bounds on quan tities of interest for problems in the XFEM context has been presented in [36]. This new technique is an extension to enriched approxima tions of the constitutive relation error (CRE) concept previously used to obtain error bounds for FEM formulations.

Ródenas et al. [37] developed a modification of the SPR technique, specifically adapted to the XFEM framework, that makes use of a singular + smooth stress field splitting technique around the crack tip similar to that described in [24] for FEM. Ref. [37] uses a simplified version of the SPR C technique [23] to enforce the boundary equi librium equation. This simplified SPR C technique imposes the fulfillment of the boundary equilibrium equation in boundary nodes but does not impose the satisfaction of the internal equilibrium equation.

\subsection{Objective and outline of the paper}

Although a few techniques to obtain upper bounds for XFEM approximations have been developed through a residual approach, to the authors' knowledge, there are no available recovery based techniques to evaluate upper error bounds in XFEM.

This paper proposes an improvement of the technique described in [31] and its adaptation to XFEM in order to obtain sharp upper bounds of the exact error in energy norm for linear elastic fracture mechanics (LEFM) problems solved with this technique.

The remainder of the paper is organized as follows: Section 2 briefly introduces the problem and presents the XFEM and the inter action integral used to evaluate the stress intensity factors (SIFs) $K_{\mathrm{I}}$ and $K_{\mathrm{II}}$. The main concepts exposed in [31] are resumed in Section 3 and serve as the basis for the development of the technique for error bounding in XFEM presented in Section 4. The numerical examples included in Section 5 demonstrate that the proposed strategy pro vides very sharp and reliable practical upper error bound estimates for XFEM. Finally, Section 6 provides a summary and some concluding remarks.

\section{Problem statement and XFEM solution}

Consider the 2D linear elasticity problem. The unknown displace ment field $\mathbf{u}$, taking values in $\Omega \subset \mathbb{R}^{2}$, is the solution of the boundary value problem

$$
\begin{aligned}
& -\nabla \cdot \boldsymbol{\sigma}(\mathbf{u})=\mathbf{b} \text { in } \Omega \\
& \boldsymbol{\sigma}(\mathbf{u}) \cdot \mathbf{n}=\mathbf{t} \text { on } \Gamma_{N} \\
& \mathbf{u}=\mathbf{0} \text { on } \Gamma_{D}
\end{aligned}
$$

where $\Gamma_{N}$ and $\Gamma_{D}$, with $\partial \Omega=\Gamma_{N} \cup \Gamma_{D}$ and $\Gamma_{N} \cap \Gamma_{D}=\varnothing$, are the Neumann and Dirichlet parts of the boundary. The Dirichlet boundary condition (1c) is taken homogeneous for the sake of simplicity.

The weak form of the problem reads: Find $\mathbf{u} \in V$ such that

$a(\mathbf{u}, \mathbf{v})=l(\mathbf{v}) \quad$ for all $\mathbf{v} \in V$

where $V$ is the standard test space for the elasticity problem and

$$
\begin{aligned}
& a(\mathbf{u}, \mathbf{v}):=\int_{\Omega} \boldsymbol{\sigma}(\mathbf{u}): \boldsymbol{\varepsilon}(\mathbf{v}) \mathrm{d} \Omega=\int_{\Omega} \boldsymbol{\sigma}(\mathbf{u}): D^{1}: \boldsymbol{\sigma}(\mathbf{v}) \mathrm{d} \Omega \\
& l(\mathbf{v}):=\int_{\Omega} \mathbf{b} \cdot \mathbf{v} \mathrm{d} \Omega+\int_{\Gamma_{N}} \mathbf{t} \cdot \mathbf{v} \mathrm{d} \Gamma
\end{aligned}
$$

where $D$ is the Hooke's tensor, $\boldsymbol{\sigma}$ and $\boldsymbol{\varepsilon}$ denote the stress and strain operators.

The bilinear form $a(\cdot, \cdot)$ can also be expressed in terms of stresses by formally introducing $\bar{a}(\cdot, \cdot)$ such that

$a(\boldsymbol{\sigma}, \boldsymbol{\tau}):=\int_{\Omega} \boldsymbol{\sigma}: D^{1}: \tau \mathrm{d} \Omega$

Note that $a(\mathbf{u}, \mathbf{v})=a(\boldsymbol{\sigma}(\mathbf{u}), \boldsymbol{\tau}(\mathbf{v}))$.

LEFM problems are characterized by the singularity at the crack tip. The following expressions show the first term of the asymptotic 
expansion of the solution for mixed mode load conditions in 2D [38]:

$$
\begin{aligned}
& \left\{\begin{array}{l}
u \\
v
\end{array}\right\}=\frac{K_{1}}{2 \mu} \sqrt{\frac{r}{2 \pi}}\left\{\begin{array}{c}
\cos \frac{\phi}{2}\left[\kappa-1+2 \sin ^{2} \frac{\phi}{2}\right] \\
\sin \frac{\phi}{2}\left[\kappa+1-2 \cos ^{2} \frac{\phi}{2}\right]
\end{array}\right\} \\
& +\frac{K_{\mathrm{II}}}{2 \mu} \sqrt{\frac{r}{2 \pi}}\left\{\begin{array}{l}
\sin \frac{\phi}{2}\left[\kappa+1+2 \cos ^{2} \frac{\phi}{2}\right] \\
-\cos \frac{\phi}{2}\left[\kappa-1-2 \sin ^{2} \frac{\phi}{2}\right]
\end{array}\right\} \\
& \left\{\begin{array}{l}
\sigma_{\text {sing }, 11} \\
\sigma_{\text {sing }, 12} \\
\sigma_{\text {sing }, 22}
\end{array}\right\}=\frac{K_{I}}{\sqrt{2} \pi r} \cos \frac{\phi}{2}\left\{\begin{array}{l}
1-\sin \frac{\phi}{2} \sin \frac{3 \phi}{2} \\
\sin \frac{\phi}{2} \cos \frac{3 \phi}{2} \\
1+\sin \frac{\phi}{2} \sin \frac{3 \phi}{2}
\end{array}\right\} \\
& +\frac{K_{I I}}{\sqrt{ } 2 \pi r}\left\{\begin{array}{l}
-\sin \frac{\phi}{2}\left[2+\cos \frac{\phi}{2} \cos \frac{3 \phi}{2}\right] \\
\cos \frac{\phi}{2}\left[1-\sin \frac{\phi}{2} \sin \frac{3 \phi}{2}\right] \\
\sin \frac{\phi}{2} \cos \frac{\phi}{2} \cos \frac{3 \phi}{2}
\end{array}\right\}
\end{aligned}
$$

where $r$ and $\phi$ represent polar coordinates with respect to the crack tip, $K_{\mathrm{I}}$ and $K_{\mathrm{II}}$ are the stress intensity factors (SIFs) for modes I and II, $\mu$ is the shear modulus, and $\kappa$ is the Kolosov constant, defined as functions of $E$ (Young's modulus) and $\nu$ (Poisson's coefficient) according to the following expressions

$$
\mu=\frac{E}{2(1+v)}, \quad \kappa= \begin{cases}3-4 v & \text { plane strain } \\ \frac{3-v}{1+v} & \text { plane stress }\end{cases}
$$

\subsection{The extended finite element method (XFEM)}

In the XFEM formulation used to solve the problem stated above, the displacement discontinuity across the crack is introduced by means of the use of additional degrees of freedom in the nodes of elements that contain the crack. This precludes the need for a conforming mesh to describe the geometry of the crack $[39,40]$. The XFEM formulation also introduces singular enrichment functions to represent the singular behaviour of the solution around the crack tip that reduce the need for the use of adaptive analysis techniques to improve the accuracy of the results. Thus, the XFEM displacements interpolation for point $\mathbf{x}$ in a $2 \mathrm{D}$ model is given by:

$\left.\mathbf{u}_{h}=\sum_{i \in I} N_{i} \mathbf{a}_{i}+\sum_{j \in J} N_{j} H(\mathbf{x}) \mathbf{b}_{j}+\sum_{m \in M} N_{m} \sum_{\ell}^{4} F_{\ell}(\mathbf{x}) \mathbf{c}_{m}^{\ell}\right)$

where $N_{i}$ is the shape function associated with node $i, \mathbf{a}_{i}, \mathbf{b}_{j}$ and $\mathbf{c}_{m}$ are nodal degrees of freedom corresponding to displacements. In the equation above, $I$ is the set of all the nodes in the mesh, $M$ is the subset of nodes enriched with crack tip functions and $J$ is the subset of nodes enriched with the Heaviside function, see Fig. 1. The Heaviside function $H(\mathbf{x})$ in Eq. (7), with unit modulus and change of sign on the crack faces, describes the discontinuity of the displacements field in elements intersected by the crack. The $F_{\ell}(\mathbf{x})$ singular functions repre sent the first term of the asymptotic expansion of the displacement

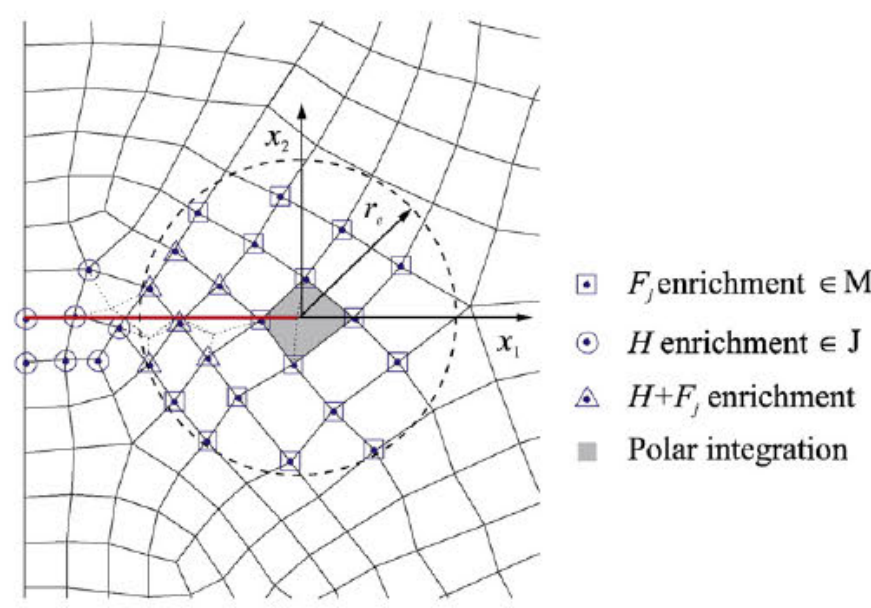

Fig. 1. Classification of nodes in XFEM. Fixed enrichment area of radius $r_{e}$,

field, see Eq. (5), in the vicinity of the crack tip. The $F_{\ell}(\mathbf{x})$ functions for the 2D case are:

$$
\left\{F_{\ell}(r, \phi)\right\} \equiv \sqrt{ } r\left\{\sin \frac{\phi}{2}, \cos \frac{\phi}{2}, \sin \frac{\phi}{2} \sin \phi, \cos \frac{\phi}{2} \sin \phi\right\} .
$$

The XFEM implementation used to obtain the numerical results presented in this paper is described in detail in [37]. Summarizing, the main characteristics of this implementation are:

- Use of bilinear quadrilaterals.

- Decomposition of elements containing the crack into integration subdomains that do not contain the crack [1].

- Use of the quasi polar integration proposed by Laborde et al. [7], with a $5 \times 5$ quadrature rule, in triangular integration subdomains of elements containing the crack tip (see Fig. 1).

- Use of a circular fixed enrichment area $B\left(x_{0}, r_{e}\right)$ with radius $r_{e}$ with its centre at the crack tip $x_{0}$ (see Fig. 1 ) as proposed in $[7,8]$ to improve the convergence rate of the XFEM solution.

\subsection{Evaluation of stress intensity factors (SIFs)}

The Stress Intensity Factors (SIFs) $K_{\mathrm{I}}$ and $K_{\mathrm{II}}$ are characterizing parameters in linear elastic fracture mechanics problems. Global methods used to extract the values of the SIFs are usually considered to be the most accurate and efficient $[41,42]$. Global methods based on the equivalent domain integral of path independent integrals (EDI methods) are especially appropriate for FEM or XFEM frameworks.

The interaction integral described in $[25,26]$ has been used in this paper. This method provides accurate values of the SIFs $K_{\mathrm{I}}$ and $K_{\mathrm{II}}$ for mixed mode problems using auxiliary extraction fields. The reader is referred to [37] and the references therein for further details.

\section{Upper bounds of the error in FEM}

The upper bound property of the error requires the recovered stress field to be statically admissible. In the technique described in [31], devoted to the evaluation of upper bounds of the error in FEM using recovery based estimates, equilibrium is obtained locally at each patch. Later, a postprocessing technique based on the Partition of Unity concept is used to enforce continuity. However, this postprocess slightly modifies the global equilibrium. As a result, the final stress field is continuous but the statical admissibility is only obtained ap proximately. The technique proposed in this reference takes into account the equilibrium defaults of the recovered stress field to 
evaluate an upper bound of the error. A summary of the theoretical basis of this technique is outlined below:

\subsection{Statically admissible stress field and upper bounds}

The basis of the technique proposed in [31] is Proposition 3. Let $\boldsymbol{\sigma}_{e}^{*}:=\boldsymbol{\sigma}^{*}-\boldsymbol{\sigma}\left(\mathbf{u}^{h}\right)$ be an error estimate of the stress field $\boldsymbol{\sigma}\left(\mathbf{u}^{h}\right)$ evaluated using FEM, where $\boldsymbol{\sigma}^{*}$ is a recovered statically admissible stress field. Let $\|\mathbf{e}\|$ be the exact error in energy norm and $\sqrt{a}\left(\boldsymbol{\sigma}_{e}^{*}, \boldsymbol{\sigma}_{e}^{*}\right)$ the energy norm associated to $\boldsymbol{\sigma}_{e}^{*}$ :

$\|\mathbf{e}\|^{2}=\int_{\Omega}\left(\boldsymbol{\sigma}-\boldsymbol{\sigma}\left(\mathbf{u}^{h}\right)\right)^{T} \mathbf{D}{ }^{1}\left(\boldsymbol{\sigma}-\boldsymbol{\sigma}\left(\mathbf{u}^{h}\right)\right) d \Omega$

$a\left(\boldsymbol{\sigma}_{e}^{*}, \boldsymbol{\sigma}_{e}^{*}\right)=\int_{\Omega}\left(\boldsymbol{\sigma}^{*}-\boldsymbol{\sigma}\left(\mathbf{u}^{h}\right)\right)^{T} \mathbf{D}^{1}\left(\boldsymbol{\sigma}^{*}-\boldsymbol{\sigma}\left(\mathbf{u}^{h}\right)\right) d \Omega$

Under these circumstances and recalling that $\mathbf{u}^{h}$ is kinematically admissible one gets:

$\|\mathbf{e}\|^{2} \leq a\left(\boldsymbol{\sigma}_{e}^{*}, \boldsymbol{\sigma}_{e}^{*}\right)$

\subsection{Nearly statically admissible stress field and upper bound of the error}

Ref. [31] proposes the use of the SPR C technique [23] to obtain interpolation polynomials $\boldsymbol{\sigma}_{i}^{*}$ that describe the recovered stress field in the patch of elements associated to each vertex node $i$. The SPR C technique ensures the satisfaction of the internal and boundary equilibrium equations and also the satisfaction of the strains compatibility equation.

Remark 1. Body loads b are approximated by a 1st order Taylor's expansion around the patch assembly node, whereas boundary tractions $\mathbf{t}$ are approximated by a 2nd order Taylor's expansion around the same point. The results obtained using this approximation are very accurate.

The global continuous recovered stress field $\boldsymbol{\sigma}^{*}$ is obtained from the local descriptions of stresses in each patch $\sigma_{i}^{*}$ using the Partition of Unity concept as indicated in the following equation:

$\boldsymbol{\sigma}^{*}(\mathbf{x})=\sum_{i}^{n_{V}} N_{i}^{\prime}(\mathbf{x}) \boldsymbol{\sigma}_{i}^{*}(\mathbf{x})$

where $n_{V}$ is the number of vertex nodes in each element and $N_{i}^{\prime}$ are the linear version of the shape function corresponding to each vertex node. This technique to obtain $\boldsymbol{\sigma}^{*}$ was termed the conjoint polynomial enhancement in 1994 by Blacker and Belytschko[21].

The divergence of $\boldsymbol{\sigma}^{*}$ is:

$-\nabla \cdot \boldsymbol{\sigma}^{*}=-\sum_{i}^{n_{V}} N_{i} \cdot \underbrace{\nabla \boldsymbol{\sigma}_{i}^{*}}_{=-\mathbf{b}}-\sum_{i}^{n_{V}} \boldsymbol{\sigma}_{i}^{*} \cdot \nabla N_{i}^{\prime}=\mathbf{b}-\sum_{i}^{n_{V}} \boldsymbol{\sigma}_{i}^{*} \cdot \nabla N_{i}^{\prime}$.

This equation shows that despite of the fact that the local representation of stresses at each patch $\boldsymbol{\sigma}_{i}^{*}$ satisfies the equilibrium equations, the $\boldsymbol{\sigma}^{*}$ stresses are not equilibrated due to the term

$\mathbf{s}=\sum_{i}^{n_{V}} \boldsymbol{\sigma}_{i}^{*} \cdot \nabla N_{i}^{\prime}$

which can be considered as an equilibrium residual resulting from the evaluation of the continuous stress field $\boldsymbol{\sigma}^{*}$ using Eq. (12).
Thus, $\boldsymbol{\sigma}^{*}$ is a nearly statically admissible stress field that satisfies the boundary equilibrium equation (each $\sigma_{i}^{*}$ satisfies this equation) but does not exactly fulfil the internal equilibrium equation. In fact, it fulfils a slightly modified version of this equation:

$-\nabla \boldsymbol{\sigma}^{*}=\mathbf{b}+\mathbf{s}$

Under these circumstances Proposition 5 in Ref. [31] provides the following expression for the upper bound of the error in energy norm

$\|\mathbf{e}\|^{2} \leq a\left(\boldsymbol{\sigma}_{e}^{*}, \boldsymbol{\sigma}_{e}^{*}\right)-2 \int_{\Omega} \mathbf{e} \cdot \mathbf{s} d \Omega$

And using the Cauchy Schwartz inequality:

$\|\mathbf{e}\|^{2} \leq a\left(\boldsymbol{\sigma}_{e}^{*}, \boldsymbol{\sigma}_{e}^{*}\right)+2\left|\int_{\Omega} \mathbf{e} \cdot \mathbf{s} d \Omega\right| \leq a\left(\boldsymbol{\sigma}_{e}^{*}, \boldsymbol{\sigma}_{e}^{*}\right)+2|\mathbf{e}|_{L_{2}}|\mathbf{s}|_{L_{2}}=E_{U B 0}^{2}$

According to Strouboulis et al. [32] we have to distinguish between a theoretical upper estimator, its computed version and a computable upper estimator. It is possible to have a theoretical upper estimator with good effectivity greater than one while its computed version can, under certain circumstances, severely underestimate the norm of the exact error (specially in the context of the GFEM, XFEM and other similar methods). By computable upper estimator the authors refer to a corrected version of the computed estimate so that it can guarantee the upper bound property.

According to the definitions given by these authors, the expression shown in Eqs. (16) and (17) are theoretical upper estimators. A procedure to obtain a computed version of Eq. (17) was presented in Ref. [31].

In Eq. (17) the terms $\overline{\mathrm{a}}\left(\boldsymbol{\sigma}_{e}^{*}, \boldsymbol{\sigma}_{e}^{*}\right)$ and $|\mathbf{s}|_{\mathrm{L}_{2}}$ are computable. Díez et al. [31] proposed a technique that can be used to obtain an accurate estimation of $|\mathbf{e}|_{\mathrm{L}_{2}}$. The convergence rate of $|\mathbf{e}|_{\mathrm{L}_{2}}$ is higher than the convergence rate of $\|\mathbf{e}\|$, therefore the use of a good approxima tion to $|\mathbf{e}|_{\mathrm{L}_{2}}$ is enough to provide good quality upper bounds for $\|\mathbf{e}\|$, as proven by the numerical examples. The technique used to estimate $|\mathbf{e}|_{\mathrm{L}_{2}}$ consists of using the last mesh of the sequence of meshes as the reference solution for the evaluation of $|\mathbf{e}|_{L_{2}}$ in the rest of the meshes. Finally, the value for the last mesh, $N$, is obtained by extrapola tion from the value calculated for mesh $N-1$. The technique provided good quality computed upper bounds for $\|\mathbf{e}\|$ in problems with smooth solutions. However, inaccurate upper bounds were obtained for problems with singular solutions as a result of the increase of the $|\mathbf{s}|_{\mathrm{L}_{2}}$ term with the mesh refinement.

\section{Upper bounds of the error in XFEM}

This section presents a new theoretical upper bound of the error in energy norm, more general than Eq. (16), and adapted for LEFM problems solved with the XFEM.

The proposed technique, as in the FEM case, is based on the evaluation of a nearly statically admissible recovered stress field $\boldsymbol{\sigma}^{*}$ and the consideration of the defects of equilibrium through the use of a correction term. For the evaluation of $\boldsymbol{\sigma}^{*}$ we propose the use of a recovery technique specially adapted to the XFEM called SPR CX, derived from the SPR technique and described in Section 4.1. Regarding to the consideration of the equilibrium defaults, and in order to obtain the computed version of the theoretical bound, we propose the use of a technique that noticeably outperforms the behaviour of the technique described in Section 3. This new tech nique, which provides very sharp upper error bounds, is presented in Section 4.2 


\subsection{The SPR CX stress recovery technique}

The SPR CX technique described here, derived from the stress recovery technique exposed in $[23,24,37]$, will be used to obtain the recovered nearly statically admissible $\boldsymbol{\sigma}^{*}$ field.

\subsubsection{Equilibrated recovered stress field at patches based on a singular + smooth stress field splitting}

The exact stress field can be expressed as the sum of two fields, a smooth stress field $\boldsymbol{\sigma}_{\text {smo }}$ and a singular stress field $\boldsymbol{\sigma}_{\text {sing }}$ :

$\boldsymbol{\sigma}=\boldsymbol{\sigma}_{\text {smo }}+\boldsymbol{\sigma}_{\text {sing }}$.

Considering the above expression, the recovered stress field $\boldsymbol{\sigma}^{*}$ required to compute the error estimate given in Eq. (10) can be expressed as the contribution of two recovered stress fields, smooth $\boldsymbol{\sigma}_{\text {smo }}^{*}$ and singular $\boldsymbol{\sigma}_{\text {sing }}^{*}$ :

$\boldsymbol{\sigma}^{*}=\boldsymbol{\sigma}_{\text {smo }}^{*}+\boldsymbol{\sigma}_{\text {sing }}^{*}$

The stress field represented by the first term of the asymptotic expansion in the vicinity of the singular point given in Eq. (6) will be used to obtain the recovered singular field $\boldsymbol{\sigma}_{\text {sing. This equation }}^{*}$ provides an accurate representation of the singular stress field using the SIF values, $K_{\mathrm{I}}^{*}$ and $K_{\mathrm{II}}^{*}$, evaluated for example using the interaction integral as suggested in this paper. Therefore, the equation used to evaluate $\boldsymbol{\sigma}_{\text {sing }}^{*}$ is:

$$
\begin{aligned}
&\left\{\begin{array}{l}
\sigma_{\text {sing, } 11}^{*} \\
\sigma_{\text {sing, }, 12}^{*} \\
\sigma_{\text {sing }, 22}^{*}
\end{array}\right\}= \frac{K_{\mathrm{I}}^{*}}{\sqrt{ } 2 \pi r} \cos \frac{\phi}{2}\left\{\begin{array}{c}
1-\sin \frac{\phi}{2} \sin \frac{3 \phi}{2} \\
\sin \frac{\phi}{2} \cos \frac{3 \phi}{2} \\
1+\sin \frac{\phi}{2} \sin \frac{3 \phi}{2}
\end{array}\right\} \\
&+\frac{K_{\mathrm{II}}^{*}}{\sqrt{ } 2 \pi r}\left\{\begin{array}{l}
-\sin \frac{\phi}{2}\left[2+\cos \frac{\phi}{2} \cos \frac{3 \phi}{2}\right] \\
\cos \frac{\phi}{2}\left[1-\sin \frac{\phi}{2} \sin \frac{3 \phi}{2}\right] \\
\sin \frac{\phi}{2} \cos \frac{\phi}{2} \cos \frac{3 \phi}{2}
\end{array}\right\}
\end{aligned}
$$

The $\boldsymbol{\sigma}^{h}$ stresses directly obtained using the XFEM for this type of problems are a finite element approximation of the stress field $\boldsymbol{\sigma}$. An FE type stress representation of the smooth field $\boldsymbol{\sigma}_{\text {smo }}^{h}$ can be obtained using the following equation:

$\boldsymbol{\sigma}_{\text {smo }}^{h}=\boldsymbol{\sigma}^{h}-\boldsymbol{\sigma}_{\text {sing }}$

Therefore, assuming that $\boldsymbol{\sigma}_{\text {sing }}^{*}$ is a good approximation of $\boldsymbol{\sigma}_{\text {sing, }}$ $\boldsymbol{\sigma}_{s m o}^{h}$ can be calculated using the following expression:

$\boldsymbol{\sigma}_{\text {smo }}^{h} \approx \boldsymbol{\sigma}^{h}-\boldsymbol{\sigma}_{\text {sing }}^{*}$

Once the $\boldsymbol{\sigma}_{\text {smo }}^{h}$ field has been obtained, the SPR C technique [23] is used to obtain an equilibrated description of the smooth solution at each patch $\boldsymbol{\sigma}_{\text {smo }}^{*}$, where $i$ indicates the $i$ th patch assembly node. Both, a discrete and a continuous approach can be used for the least squares fitting[43]. In the discrete formulation, which is the traditional approach, the stresses are sampled at the superconvergence points. In the continuous formulation, the functional representing the $L_{2}$ norm of the difference between the XFEM (or FEM) solution gradient $\boldsymbol{\sigma}^{h}$ and the polynomial expansion representing the recovered field $\boldsymbol{\sigma}^{*}$ is minimized. This procedure implies sampling the stresses at the integration points of the quadrature rule but also the use of the weighting factors given by the quadrature rule at each of these points. Our numerical experience has shown a better performance of the error estimator using the continuous formulation, which has been the approach used in our implementation.

Finally, the recovered stresses at each patch are evaluated as

$\boldsymbol{\sigma}_{i}^{*}=\boldsymbol{\sigma}_{s m o_{i}}^{*}+\boldsymbol{\sigma}_{\text {sing }}^{*}$

This decomposition recovery technique is particularly effective in the vicinity of the singularity, although it does not need to be used in the whole domain of the problem. Far from the singularity, the equilibrated stress field at each patch can be adequately recovered using the SPR C technique. In the proposed procedure, if the patch contains an enriched node, the singular + smooth stress decomposi tion method, described above, is used to compute $\boldsymbol{\sigma}_{i}^{*}$, otherwise the SPR C technique is used to directly obtain $\boldsymbol{\sigma}_{i}^{*}$ from $\boldsymbol{\sigma}^{h}$. Thus, in patches outside the decomposition area $\boldsymbol{\sigma}_{i}^{*}$ are stress interpolation polynomials, whereas in patches within the decomposition area $\boldsymbol{\sigma}_{i}^{*}$ represents stress interpolation functions that contain a polynomial part $\boldsymbol{\sigma}_{\text {smo }}^{*}$ and a singular part $\boldsymbol{\sigma}_{\text {sing. }}^{*}$.

Fig. 2 illustrates how the patch stress interpolation functions $\boldsymbol{\sigma}_{i}^{*}$ are obtained in the different domain areas of the problem.

Remark 2. The recovered stress field at each patch $\boldsymbol{\sigma}_{i}^{*}$ is obtained by the addition of two functions that, individually, satisfy the equilibrium equations. On the one hand the SPR C technique enforces $\boldsymbol{\sigma}_{s m o_{i}}^{*}$ to satisfy the internal equilibrium and boundary equilibrium equations; on the other hand $\boldsymbol{\sigma}_{\text {sing }}^{*}$ also satisfies the internal equilibrium equation because it is evaluated using Eq. (20), derived from Eq. (6) which is the first term of the asymptotic expansion that describes the stress field in the vicinity of the crack tip. Therefore, adding these two terms produces a statically admissible stress field.

Remark 3. Using the argument exposed in Remark 2 we can also justify that $\boldsymbol{\sigma}_{i}^{*}$ also satisfies the compatibility equation. Note that the

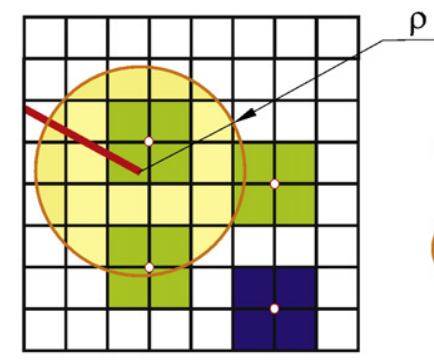

Patch within decomposition area

Crack

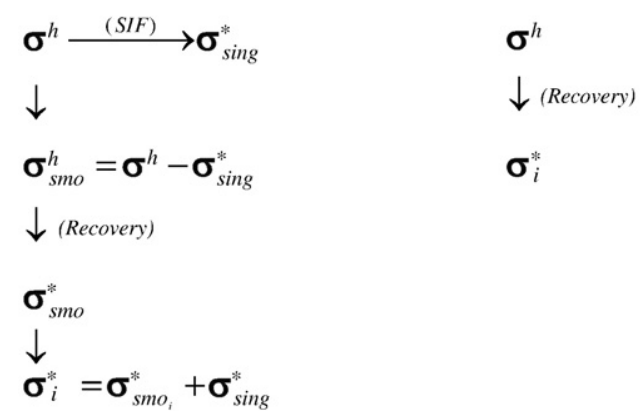

Fig. 2. Calculation of $\boldsymbol{\sigma}_{i}^{*}$ in different types of patches. 
satisfaction of this equation is not necessary for the upper bound property. Thus, it is only used to improve the quality of $\boldsymbol{\sigma}^{*}$.

Remark 4. Observe that the use of this technique can be directly extended to deal with problems with multiple crack tips, as long as they do not interact with each other or with the boundary, because the singular + smooth stress field decomposition technique is only used in the vicinity of the crack tip.

\subsubsection{Patch assembly}

As in the standard SPR technique, the patches are composed by the elements that surround each of the vertex nodes of the mesh. However, for patches containing the crack it is not convenient to use one single function $\boldsymbol{\sigma}_{i}^{*}$ to represent the stress state in the whole patch. In these cases two different patches are created, one at each side of the crack. Each of these two patches is composed by the integration subdomains of the elements of the original patch of elements located at each side of the crack. This allows the use of different functions to represent the stress state at each side of the crack.

In the SPR CX technique crack surfaces are treated as any other external boundary. Assuming that there is no contact between the crack surfaces, these surfaces will be free of external tractions. The procedure used to obtain $\boldsymbol{\sigma}_{i}^{*}$ in patches located along the boundary of the domain is described in [23]. According to this procedure, the boundary equilibrium equation can be easily imposed along the external boundaries. To do this we will use a local Cartesian reference system to describe $\boldsymbol{\sigma}_{i}^{*}$ with the origin located over the patch assembly node and with the axes aligned with the normal and tangential direction with respect to the surface. This procedure cannot be directly applied on the crack because in the general case there are no nodes along the crack. In this case, the local Cartesian reference system used to describe $\boldsymbol{\sigma}_{i}^{*}$ will be located on the point of the crack (or its prolongation) closest to the patch assembly node, as described in Fig. 3.

Remark 5. Some of the subpatches next to the crack can even be composed by a single integration domain. Rank deficiency during the least squares fitting is avoided in these cases if the number of Gauss points used in the integration subdomains is equal to or greater than the number of polynomial terms used to describe each stress component.

4.1.3. Nearly statically admissible continuous recovered stress field $\boldsymbol{\sigma}^{*}$

The stress values at each point into the elements are evaluated from different patches because patches overlap each other. More precisely, the stress values at each point into the elements are evaluated from $n_{v}$ patches, being $n_{v}$ the number of vertex nodes in the element. As in the

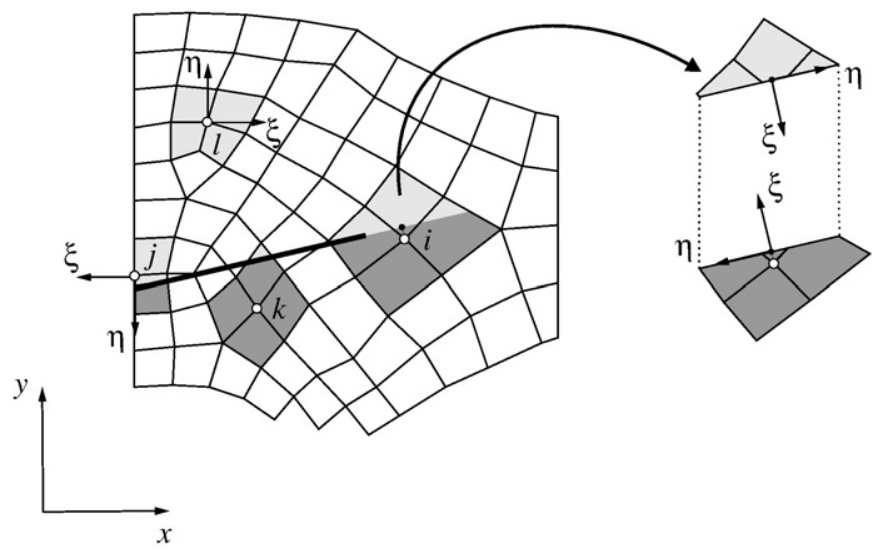

Fig. 3. Reference systems used in the evaluation of $\boldsymbol{\sigma}_{i}^{*}$ on external boundaries and crack surfaces.
FEM context, a continuous recovered stress field $\boldsymbol{\sigma}^{*}$ will be evaluated from the stress contribution corresponding to each patch $\sigma_{i}^{*}$ by means of the Partition of Unity procedure indicated in Eq. (12).

Again, in the XFEM context this process introduces defects in the equilibrium and compatibility equations. These lacks of equilibrium will be considered in the evaluation of the upper bound of the discretization error.

\subsection{Correction of the estimated error in energy norm: The theoretical} upper bound of the error.

The technique previously described provides a field $\boldsymbol{\sigma}^{*}$ that does not fully satisfy the internal equilibrium equation, but verifies the slightly modified version of this equation shown in Eq. (15).

Remark 1 exposed that with the SPR C technique [24] body loads b are approximated by a 1st order Taylor's expansion around the patch assembly node, whereas boundary tractions $\mathbf{t}$ are approximated by a 2nd order Taylor's expansion. In the examples presented in Section 5 body loads are assumed to be null, therefore, the approximation does not introduce further equilibrium defects in the internal equilibrium equation. The second assumption related to the approximation of $\mathbf{t}$ using a 2nd order Taylor's expansion around the patch assembly node can be considered as very accurate in the FEM framework. However, the lack of equilibrium along the boundary must also be taken under consideration in XFEM, where the size of the elements tends to be bigger than in FEM,

Proposition 1. Let $\boldsymbol{\sigma}^{*}$ be such that $\boldsymbol{\sigma}^{*} \cdot \mathbf{n}$ is continuous almost every where (a.e.) along any interior curve $\Gamma \subset \Omega$ (being $\mathbf{n}$ the unit normal to $\Gamma$ ) and

$$
\begin{aligned}
& -\nabla \boldsymbol{\sigma}^{*}=\mathbf{b}+\mathbf{s} \quad \text { a.e. in } \Omega \\
& \boldsymbol{\sigma}^{*} \cdot \mathbf{n}=\mathbf{t}+\mathbf{r} \quad \text { a.e. on in } \Gamma_{N} .
\end{aligned}
$$

Note that if $\mathbf{s}=0$ and $\mathbf{r}=0, \boldsymbol{\sigma}^{*}$ is statically admissible. For small $\mathbf{s}$ and $\mathbf{r}$ (when compared to $\mathbf{b}$ and $\mathbf{t}$, respectively) $\boldsymbol{\sigma}^{*}$ is said to be nearly statically admissible, up to the equilibrium default terms.

Then, the following expression holds:

$a\left(\boldsymbol{\sigma}^{*}, \boldsymbol{\sigma}(\mathbf{v})\right)=l(\mathbf{v})+\int_{\Omega} \mathbf{v} \cdot \mathbf{s} d \Omega+\int_{\Gamma_{N}} \mathbf{v} \cdot \mathbf{r} d \Gamma \quad$ for all $\mathbf{v} \in V$

and, as a direct consequence assuming that the Dirichlet boundary conditions are homogeneous

$$
\|\mathbf{u}\|^{2}=a(\boldsymbol{\sigma}(\mathbf{u}), \boldsymbol{\sigma}(\mathbf{u})) \leq a\left(\boldsymbol{\sigma}^{*}, \boldsymbol{\sigma}^{*}\right)-2 \int_{\Omega} \mathbf{u} \cdot \mathbf{s} d \Omega-2 \int_{\Gamma_{N}} \mathbf{u} \cdot \mathbf{r} d \Gamma
$$

Moreover, the error approximation $\boldsymbol{\sigma}_{e}^{*}:=\boldsymbol{\sigma}^{*}-\boldsymbol{\sigma}\left(\mathbf{u}^{H}\right)$ is also providing an upper bound of the error norm, up to the equilibration default terms

$\|\mathbf{e}\|^{2}=a(\mathbf{e}, \mathbf{e})=a\left(\boldsymbol{\sigma}_{e}, \boldsymbol{\sigma}_{e}\right) \leq a\left(\boldsymbol{\sigma}_{e}{ }^{*}, \boldsymbol{\sigma}_{e}{ }^{*}\right) \quad 2 \int_{\Omega} \mathbf{e} \cdot \mathbf{s} d \Omega \quad 2 \int_{\Gamma_{N}} \mathbf{e} \cdot \mathbf{r} d \Gamma=E_{U B}^{2}$

and this latter property stands also for non homogeneous Dirichlet boundary conditions, up to oscillation terms.

These results are valid even if $\boldsymbol{\sigma}$ exhibits singularities. Note however that in the presence of singularities, the equilibrium default associated with a post processed solution $\sigma^{*}$ is possibly large unless $\boldsymbol{\sigma}^{*}$ has a singularity of the same kind.

Proof. The expression (25) is derived by the standard weighted residuals technique combined with the usual integration by parts 
from Eq. (24) (using Eqs. (24a) and (24b) and the fact that the test function $\mathbf{v}$ vanishes on $\Gamma_{D}$ ).

$$
\begin{aligned}
\int_{\Omega} \mathbf{v} \cdot(\mathbf{b}+\mathbf{s}) d \Omega & =-\int_{\Omega} \mathbf{v} \cdot \nabla \cdot \boldsymbol{\sigma}^{*} d \Omega \\
& =-\int_{\Omega} \nabla \cdot\left(\boldsymbol{\sigma}^{*} \cdot \mathbf{v}\right) d \Omega+\int_{\Omega} \nabla \mathbf{v}: \boldsymbol{\sigma}^{*} d \Omega \\
& =-\int_{\partial \Omega} \mathbf{n} \cdot\left(\boldsymbol{\sigma}^{*} \cdot \mathbf{v}\right) d \Gamma+\int_{\Omega} \frac{1}{2}\left(\nabla \mathbf{v}+\nabla^{T} \mathbf{v}\right) \cdot \boldsymbol{\sigma}^{*} d \Omega \\
& =-\int_{\Gamma_{N}} \mathbf{v} \cdot\left(\boldsymbol{\sigma}^{*} \cdot \mathbf{n}\right) d \Gamma+a\left(\boldsymbol{\sigma}^{*}, \boldsymbol{\sigma}(\mathbf{v})\right) \\
& =-\int_{\Gamma_{N}} \mathbf{v} \cdot(\mathbf{t}+\mathbf{r}) d \Gamma+a\left(\boldsymbol{\sigma}^{*}, \boldsymbol{\sigma}(\mathbf{v})\right)
\end{aligned}
$$

Then Eq. (25) follows by considering the definition of $l(\mathbf{v})$.

Thus, the proof of Eq. (26) is straightforward by considering in Eq. (25) $\mathbf{v}=\mathbf{u}$ (this requires that in the original problem, the Dirichlet boundary conditions state that $\mathbf{u}=0$ on $\Gamma_{D}$ ):

$$
\begin{aligned}
a\left(\boldsymbol{\sigma}^{*}, \boldsymbol{\sigma}(\mathbf{u})\right) & =l(\mathbf{u})+\int_{\Omega} \mathbf{u} \cdot \mathbf{s} d \Omega+\int_{\Gamma_{N}} \mathbf{u} \cdot \mathbf{r} d \Gamma \\
& =a(\boldsymbol{\sigma}(\mathbf{u}), \boldsymbol{\sigma}(\mathbf{u}))+\int_{\Omega} \mathbf{u} \cdot \mathbf{s} d \Omega+\int_{\Gamma_{N}} \mathbf{u} \cdot \mathbf{r} d \Gamma
\end{aligned}
$$

and therefore

$$
\begin{aligned}
0 \leq a\left(\boldsymbol{\sigma}(\mathbf{u})-\boldsymbol{\sigma}^{*}, \boldsymbol{\sigma}(\mathbf{u})-\boldsymbol{\sigma}^{*}\right)= & a(\boldsymbol{\sigma}(\mathbf{u}), \boldsymbol{\sigma}(\mathbf{u}))-2 a\left(\boldsymbol{\sigma}^{*}, \boldsymbol{\sigma}(\mathbf{u})\right) \\
& +a\left(\boldsymbol{\sigma}^{*}, \boldsymbol{\sigma}^{*}\right)=a(\boldsymbol{\sigma}(\mathbf{u}), \boldsymbol{\sigma}(\mathbf{u})) \\
& -2 \int_{\Omega} \mathbf{u} \cdot \mathbf{s} d \Omega-2 \int_{\Gamma_{N}} \mathbf{u} \cdot \mathbf{r} d \Gamma+a\left(\boldsymbol{\sigma}^{*}, \boldsymbol{\sigma}^{*}\right) .
\end{aligned}
$$

The proof of Eq. (27) is similar, by considering in Eq. (25) $\mathbf{v}=\mathbf{e}$ (this can be done, up to oscillation terms, even if in the original problem the Dirichlet boundary conditions are non homogeneous). Thus,

$$
\begin{aligned}
a\left(\boldsymbol{\sigma}^{*}, \boldsymbol{\sigma}(\mathbf{e})\right) & =l(\mathbf{e})+\int_{\Omega} \mathbf{e} \cdot \mathbf{s} d \Omega+\int_{\Gamma_{N}} \mathbf{e} \cdot \mathbf{r} d \Gamma \\
& =a(\boldsymbol{\sigma}(\mathbf{e}), \boldsymbol{\sigma}(\mathbf{e}))+a\left(\boldsymbol{\sigma}\left(\mathbf{u}^{h}\right), \boldsymbol{\sigma}(\mathbf{e})\right)+\int_{\Omega} \mathbf{e} \cdot \mathbf{s} d \Omega+\int_{\Gamma_{N}} \mathbf{e} \cdot \mathbf{r} d \Gamma
\end{aligned}
$$

that results on

$$
a\left(\boldsymbol{\sigma}_{e}^{*}, \boldsymbol{\sigma}(\mathbf{e})\right)=a(\boldsymbol{\sigma}(\mathbf{e}), \boldsymbol{\sigma}(\mathbf{e}))+\int_{\Omega} \mathbf{e} \cdot \mathbf{s} d \Omega+\int_{\Gamma_{N}} \mathbf{e} \cdot \mathbf{r} d \Gamma
$$

Note that the following error representation has been used

$a(\boldsymbol{\sigma}(\mathbf{e}), \boldsymbol{\sigma}(\mathbf{e}))=l(\mathbf{e})-a\left(\boldsymbol{\sigma}\left(\mathbf{u}^{h}\right), \boldsymbol{\sigma}(\mathbf{e})\right)$.

Thus, Eq. (27) is proved using the same idea as before by simply considering the positiveness of $\bar{a}\left(\boldsymbol{\sigma}(\mathbf{e})-\boldsymbol{\sigma}_{e}^{*}, \boldsymbol{\sigma}(\mathbf{e})-\boldsymbol{\sigma}_{e}^{*}\right)$.

The expression shown in Eq. (27) is a theoretical upper estimator. Its computed version remains to be calculated.

In Eq. (27) the value of $\mathbf{s}$ is evaluated using Eq. (14). On the other hand, the value of $\mathbf{r}$, that represents the residual of the boundary equilibrium equation, can be evaluated using

$\mathbf{r}=\boldsymbol{\sigma}^{*} \cdot \mathbf{n}-\mathbf{t}=\sum_{i}^{n}\left(N_{i}^{\prime} \boldsymbol{\sigma}_{i}^{*}\right) \cdot \mathbf{n}-\mathbf{t}=\sum_{i}^{n} N_{i}^{\prime}\left(\boldsymbol{\sigma}_{i}^{*} \cdot \mathbf{n}-\mathbf{t}\right)$.

Díez et al. [31] propose the evaluation of the upper bound of the error in energy norm by using Eq. (17), based in the use of the Cauchy Schwartz inequality and the evaluation of the $L_{2}$ norms of $\mathbf{e}$ and $\mathbf{s}$. Here, we propose the direct use of Eq. (27) to obtain sharper bounds of the error avoiding the use of the Cauchy Schwartz inequality, which introduces a too pessimistic approximation, as it is shown in the numerical examples. Eq. (27) requires the value of the error $\mathbf{e}$ at any point of the mesh. A procedure to obtain an approximation of $\mathbf{e}$ is presented below.

The displacement field $\mathbf{u}_{(N)}^{H}$ obtained as the solution from the last mesh of the refinement process can be considered as an approxima tion to the exact solution of the problem, $\mathbf{u} \approx \mathbf{u}_{(N)}^{H}$. This allows the evaluation of an approximation of $\mathbf{e}$ for the first $N-1$ meshes of the sequence:

$\mathbf{e}_{(i)}=\mathbf{u}-\mathbf{u}_{(i)}^{h} \approx \mathbf{u}_{(N)}^{h}-\mathbf{u}_{(i)}^{h}=\mathbf{e}_{e s(i)}$.

The use of Eq. (29) in the evaluation of $\mathbf{e}_{e s(i)}, i=1, \ldots, N-1$, requires the projection of the displacements field of mesh $N$ on mesh $i$. Finally, it is possible to obtain an estimate of integrals $-2 \int_{\Omega} \mathbf{e} \cdot \mathbf{s d} \Omega$ and $-2 \int_{\Gamma} \mathbf{e} \cdot \mathbf{r d} \Gamma$ for mesh $N$ considering the values of these integrals evaluated for the previous meshes and the use of the Richardson extrapolation technique.

As the evaluation of $\mathbf{e}_{e s}$ introduces an approximation, the results obtained using Eq. (27) cannot be taken as guaranteed upper bounds. However, Section 6 will show that the technique used to estimate $\mathbf{e}$ at each mesh provides very accurate approximations of the correction

1.- Use the SPR-CX technique to compute a continuous nearly-statically admissible field $\sigma$

- Compute the recovered singular stress field $\boldsymbol{\sigma}_{\text {sing }}^{*}$

- Compute $K_{\mathrm{I}}^{*}$ and $K_{\mathrm{II}}{ }^{*}$ using the interaction integral.

- Compute $\boldsymbol{\sigma}_{\text {sing }}^{*}$ using (20).

- Define the singular + smooth stress decomposition area $\boldsymbol{\sigma}_{i}^{*}$

- Compute $\sigma_{i}^{*}$ at each patch into the decomposition area

- Compute an FE-type representation of the smooth part: $\boldsymbol{\sigma}_{s m o}^{h} \approx \boldsymbol{\sigma}^{h}-\boldsymbol{\sigma}_{s i n g}^{*}$

- Use the SPR-C technique to compute $\boldsymbol{\sigma}_{\text {smo }_{i}}^{*}$

- Compute $\boldsymbol{\sigma}_{i}^{*}=\boldsymbol{\sigma}_{\text {smo }}^{*}+\boldsymbol{\sigma}_{\text {sing }}^{*}$

- Compute $\sigma_{i}^{*}$ at each patch outside the decomposition area using the SPR-C technique

- Compute a continuous nearly-statically admissible field $\sigma^{*}=\sum_{i=1}^{n_{i}} N_{i}^{\prime} \boldsymbol{\sigma}_{i}^{*}$

2.- Compute equilibrium defects $\mathbf{s}$ and $\mathbf{r}$ using (14) and (28).

3.- Compute $E_{S P R-C X}=\bar{a}\left(\boldsymbol{\sigma}_{e}^{*}, \boldsymbol{\sigma}_{e}^{*}\right)$

4.- Use procedure described at the end of section 4.2 to evaluate $\mathbf{e}_{e s}$ as an estimate of $\mathbf{e}$.

5.- $\quad$ Compute the error upper bound $E_{U B}=\sqrt{\bar{a}\left(\boldsymbol{\sigma}_{e}^{*}, \boldsymbol{\sigma}_{e}^{*}\right)-2 \int_{\Omega} \mathbf{e} \cdot \mathbf{s} d \Omega-2 \int_{\Gamma_{N}} \mathbf{e} \cdot \mathbf{r} d \Gamma}$

Fig. 4. Procedure for the evaluation of the upper bound of the error in energy norm. 


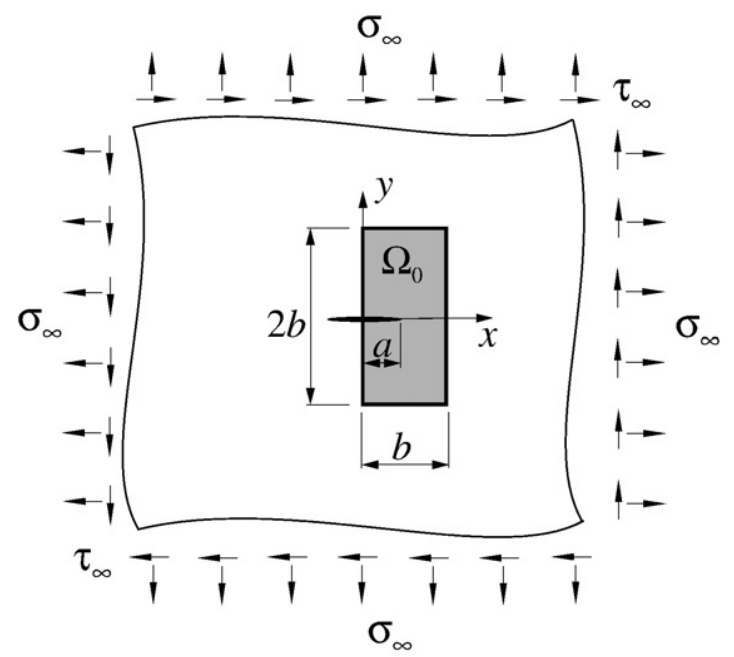

Fig. 5. Westergaard problem. Infinite plate with a crack of length $2 a$ subjected to uniform tractions $\sigma_{\infty}$ (biaxial) and $\tau_{\infty}$. Finite portion of the domain, $\Omega_{0}$, modelled with FE.

terms $-2 \int_{\Omega} \mathbf{e} \cdot \mathbf{s d} \Omega$ and $-2 \int_{\Gamma_{N}} \mathbf{e} \cdot \mathbf{r d} \Gamma$, thus validating the use of the technique proposed to obtain $\mathbf{e}$.

\subsection{Proposed procedure}

The techniques for the evaluation of the upper bound of the error in energy norm introduced above are summarized in Fig. 4.

\section{Numerical results}

The numerical analyses performed to test the behaviour of the pro posed technique are presented in this section. The classic Westergaard problem [44] has been used in the analyses as it is one of the few LEFM problems in mixed mode that has an exact analytical solution.

Bilinear quadrilaterals have been used in the numerical examples. Linear polynomials have been used in the implementation of the stress recovery technique for patches of nodes located in the interior of the domain whereas quadratic polynomials have been considered along the boundary of the domain and along the crack surfaces.

\subsection{Test problem: the Westergaard problem}

The Westergaard problem consists of an infinite plate loaded with biaxial tractions $\sigma_{x \infty}=\sigma_{y \infty}=\sigma_{\infty}$ and $\tau_{\infty}$ in the infinite, with a crack of a finite length $2 a$, as is shown in Fig. 5. Combining the externally applied tractions one can generate stress states in pure modes I or II, or in mixed mode [37].

A finite portion of the domain ( $a=1$ and $b=4$ in Fig. 5) was included in the numerical model and the projection of the stresses corresponding to the analytical Westergaard solution for modes I and II, given by the expressions below, were applied to the boundary [44].

Mode I.

$$
\begin{aligned}
\sigma_{x}^{I} & =\frac{\sigma_{\infty}}{\sqrt{ }|t|}\left[\left(x \cos \frac{\phi}{2}-y \sin \frac{\phi}{2}\right)+y \frac{a^{2}}{|t|^{2}}\left(m \sin \frac{\phi}{2}-n \cos \frac{\phi}{2}\right)\right] \\
\sigma_{y}^{I} & =\frac{\sigma_{\infty}}{\sqrt{ }|t|}\left[\left(x \cos \frac{\phi}{2}-y \sin \frac{\phi}{2}\right)-y \frac{a^{2}}{|t|^{2}}\left(m \sin \frac{\phi}{2}-n \cos \frac{\phi}{2}\right)\right] \\
\tau_{x y}^{I} & =y \frac{a^{2} \sigma_{\infty}}{|t|^{2} \sqrt{ }|t|}\left(m \cos \frac{\phi}{2}+n \sin \frac{\phi}{2}\right) .
\end{aligned}
$$

\section{Mode II.}

$$
\begin{aligned}
& \sigma_{x}^{I I}=\frac{\tau_{\infty}}{\sqrt{ }|t|}\left[2\left(y \cos \frac{\phi}{2}+x \sin \frac{\phi}{2}\right)-y \frac{a^{2}}{|t|^{2}}\left(m \cos \frac{\phi}{2}+n \sin \frac{\phi}{2}\right)\right] \\
& \sigma_{y}^{I I}=y \frac{a^{2} \tau_{\infty}}{|t|^{2} \sqrt{ }|t|}\left(m \cos \frac{\phi}{2}+n \sin \frac{\phi}{2}\right) \\
& \tau_{x y}^{I I}=\frac{\tau_{\infty}}{\sqrt{ }|t|}\left[\left(x \cos \frac{\phi}{2}-y \sin \frac{\phi}{2}\right)+y \frac{a^{2}}{|t|^{2}}\left(m \sin \frac{\phi}{2}-n \cos \frac{\phi}{2}\right)\right] .
\end{aligned}
$$

In the above equations, the stress fields are expressed as a function of the coordinates $x$ and $y$, whose origin is in the centre of the crack, where $t, m, n$ and $\phi$ are defined as:

$$
\begin{aligned}
& t=z^{2}-a^{2}=(x+i y)^{2}-a^{2}=\left(x^{2}-y^{2}-a^{2}\right)+i(2 x y)=m+i n \\
& m=\operatorname{Re} t=\operatorname{Re}\left(z^{2}-a^{2}\right)=x^{2}-y^{2}-a^{2} \\
& n=\operatorname{Im} t=\left(z^{2}-a^{2}\right)=2 x y \\
& \phi=\arg t=\arg (m-i n) \text { with } \phi \in[-\pi, \pi] .
\end{aligned}
$$

The exact SIF values for this problem are defined as:

$$
K_{\text {Iex }}=\sigma_{\infty} \sqrt{ } \pi a \quad K_{\text {Ilex }}=\tau_{\infty} \sqrt{ } \pi a .
$$

Bilinear elements with a smooth + singular decomposition area of a radius $\rho=0.5$ equal to the radius $r_{e}$ of the fixed enrichment area have

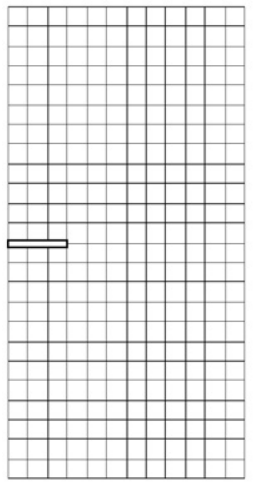

Mesh 1

288 elem.

723 dof

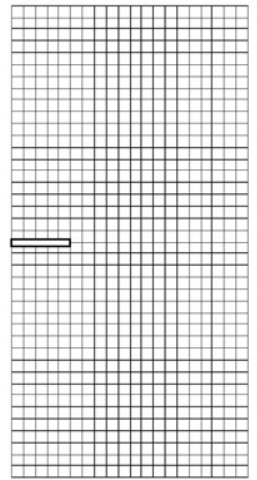

Mesh 2

800 elem.

1895 dof

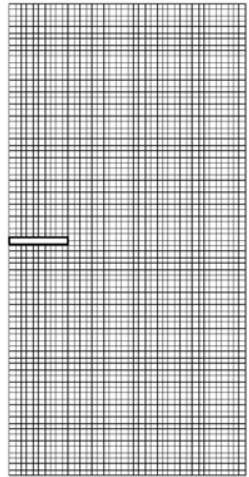

Mesh 3

3200 elem.

7289 dof

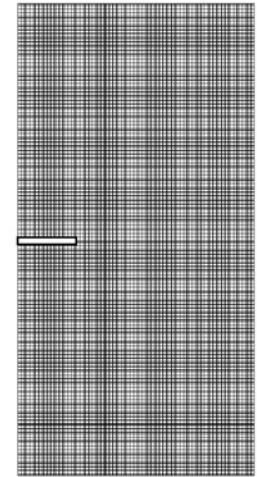

Mesh 4

12800 elem

28637 dof

Fig. 6. Sequence of uniform meshes. 


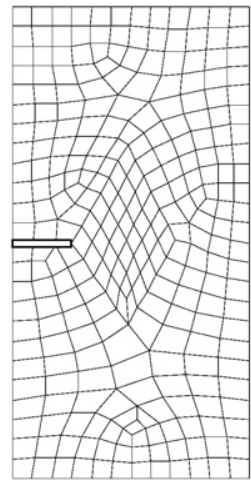

Mesh 1

273 elem.

689 dof

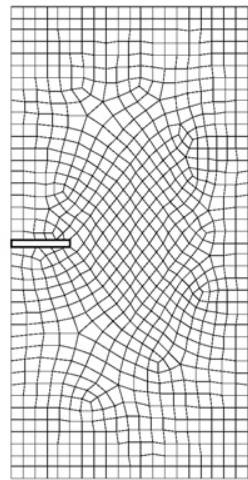

Mesh 2

818 elem.

1907 dof

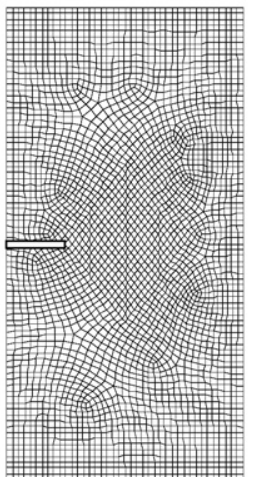

Mesh 3

3272 elem.

6779 dof

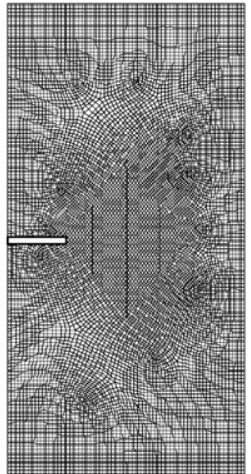

Mesh 4

13088 elem.

28767 dof

Fig. 7. Sequence of non-uniform meshes.
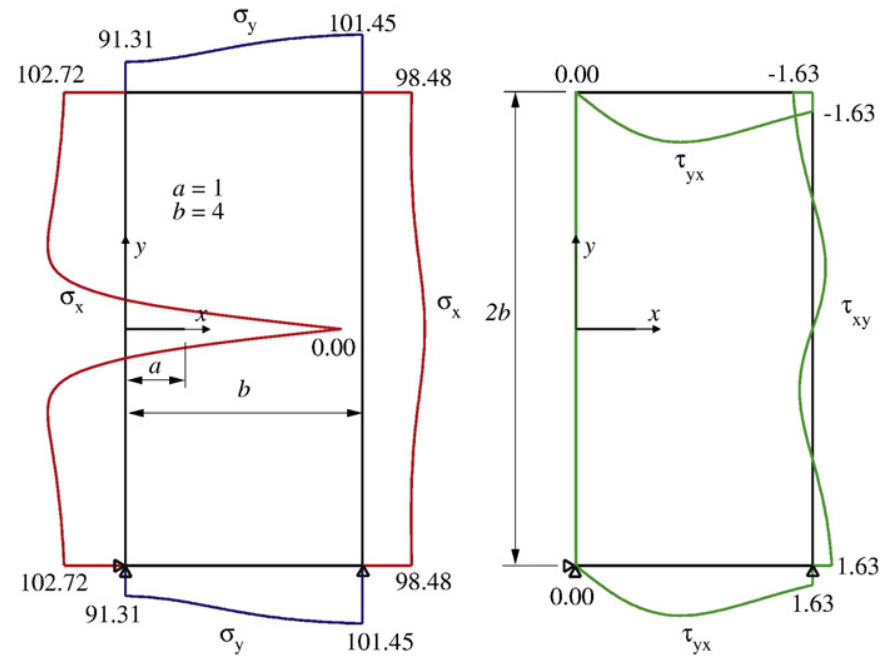

Fig. 8. Mode I. Model for crack in infinite plate subjected to biaxial traction in the infinite. $\left(\sigma_{\infty}=100, \tau_{\infty}=0\right) . K_{I, e x}=177.2453850905516$.
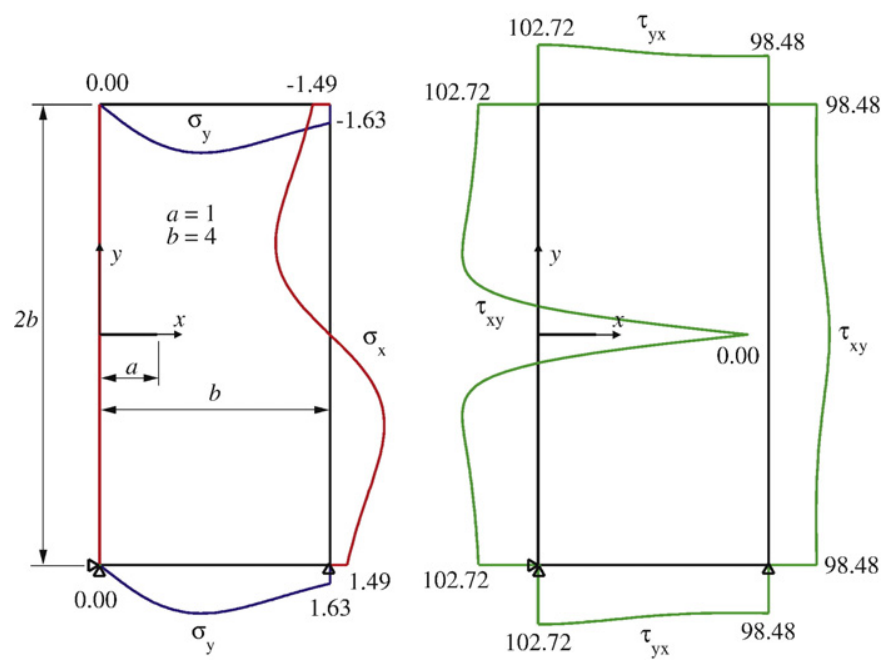

Fig. 9. Mode II. Model for crack in infinite plate subjected to tangential stresses in the infinite. $\left(\sigma_{\infty}=0, \tau_{\infty}=100\right) . K_{I I, e x}=177.2453850905516$. been considered in the numerical analyses. Young's modulus was $E=10^{7}$, and Poisson's ratio $\nu=0.333$.

The sequences of meshes shown in Figs. 6 and 7 have been used in the numerical examples.

Three cases of the Westergaard problem were considered: pure mode I, pure mode II, and mixed mode. The geometric models and boundary conditions considering mode I, mode II and mixed mode are shown in Figs. 810.

Various approaches for the evaluation of the upper bounds of the error in energy norm have been implemented based on Eq. (27). The first one is the adaptation to XFEM of technique developed by Díez et al. [31] given by Eq. (17), which has been shown below for convenience:

$\|\mathbf{e}\|^{2} \leq a\left(\boldsymbol{\sigma}_{e}^{*}, \boldsymbol{\sigma}_{e}^{*}\right)+2\left|\int_{\Omega} \mathbf{e} \cdot \mathbf{s} d \Omega\right| \leq a\left(\boldsymbol{\sigma}_{e}^{*}, \boldsymbol{\sigma}_{e}^{*}\right)+2|\mathbf{e}|_{L_{2}}|\mathbf{s}|_{L_{2}}=E_{U B 0}^{2}$

The second technique to be implemented is the technique proposed in this paper for the evaluation of the error in energy norm as described by Eq. (27), which is also shown here for convenience:

$\|\mathbf{e}\|^{2} \leq a\left(\boldsymbol{\sigma}_{e}^{*}, \boldsymbol{\sigma}_{e}^{*}\right)-2 \int_{\Omega} \mathbf{e} \cdot \mathbf{s} d \Omega-2 \int_{\Gamma_{N}} \mathbf{e} \cdot \mathbf{r} d \Gamma=E_{U B}^{2}$
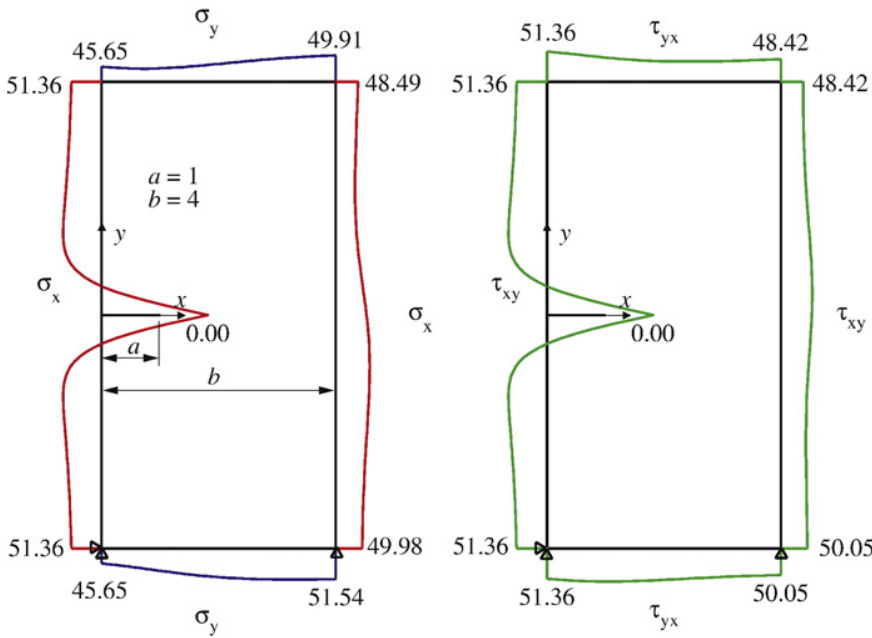

Fig. 10. Mixed Mode. Model for crack in infinite plate subjected to biaxial traction in the infinite. $\left(\sigma_{\infty}=50, \tau_{\infty}=50\right) . K_{I, e x}=K_{I I, e x}=88.6226925452758$ 


\subsection{Accuracy of the recovered stresses $\boldsymbol{\sigma}^{*}$}

The technique proposed for the computation of the upper bound of the error in energy norm is based on the evaluation of a highly accurate recovered stress field $\boldsymbol{\sigma}^{*}$. Fig. 11 shows the exact errors $\boldsymbol{\sigma}^{e x}-\boldsymbol{\sigma}^{h}$ and $\boldsymbol{\sigma}^{e x}-\boldsymbol{\sigma}^{*}$ for each of the stress components $\sigma_{x x}, \sigma_{y y}, \sigma_{x y}$ and for the von Mises stress $\sigma_{v m}$. It can be clearly observed that $\boldsymbol{\sigma}^{*}$ is, approximately, one order of magnitude more precise than the raw XFEM solution. The circle shown in the graphs represents the contour of the enrichment area that coincides with the radius of the singular + smooth splitting area.

The graphs shown in Fig. 11 represent the values of the error at the integration points in the second uniform mesh with 800 elements. Fig. 12 represents the results for a more general case considering mixed mode and a non uniform mesh of 818 elements. To obtain these graphs, the value at each integration point has been represented as a constant value in a prescribed area surrounding each integration point.

Fig. 11 shows that $\sigma^{*}$ is less accurate in the crack front. This is not a surprising result because of the proximity of the crack tip. However, the lack of accuracy is also strengthened by the process followed to impose the satisfaction of the boundary equilibrium. At each patch, $\boldsymbol{\sigma}^{*}$ is forced to satisfy the boundary equilibrium Eq. (1b) along $\xi=0$ (see Fig. 3). For patches containing the crack tip, this implies forcing $\sigma_{\xi \xi}^{*}=0$ and $\sigma_{\xi \eta}^{*}=0$, not only along the crack surface but also along the prolongation of the crack located after the crack tip (see patch for node $i$ in Fig. 3). Although the accuracy of $\boldsymbol{\sigma}^{*}$ is reduced, the use of this procedure is necessary to impose the satisfaction of the boundary equilibrium equation required to obtain the upper bound property.

Fig. 13 shows the evolution of the exact error of the solution provided by XFEM $\left\|\mathbf{u}-\mathbf{u}^{h}\right\|=\|\mathbf{e}\|$ and that corresponding to the recovered solution $\left\|\mathbf{u}-\mathbf{u}^{*}\right\|=\left\|\mathbf{e}^{*}\right\|$. The first conclusion extracted from this figure is that $\boldsymbol{\sigma}^{*}$ is more accurate than $\boldsymbol{\sigma}^{h}$ in all the analyzed examples. Furthermore, Fig. 13 shows that the rate of convergence in $\left\|\mathbf{e}^{*}\right\|$ is higher than the rate of convergence in $\|\mathbf{e}\|$, due to the high accuracy of $\sigma^{*}$. According to Zienkiewicz and Zhu[16] this proves that the error estimator $\bar{a}\left(\boldsymbol{\sigma}_{e}^{*}, \boldsymbol{\sigma}_{e}^{*}\right)$ is asymptotically exact.

\subsection{Accuracy of the upper bound $E_{U B O}$}

Fig. 14 shows the evolution of the global effectivity index $\theta$ defined as

$$
\theta=\frac{\left\|\mathbf{e}_{e s}\right\|}{\|\mathbf{e}\|}
$$

considering Mode I for the error estimator $E_{S P R-C X}$ and for the bound $E_{U В O}$. The results regarding the accuracy of these errors are similar to those shown by Díez et al. [31] using the FEM. Note that as the $\sigma^{*}$ field used to evaluate the $E_{S P R-C X}$ estimate does not fully satisfies the equilibrium requirements this estimate does not provide upper error bounds, as can be seen in Fig. 14 and more clearly in Fig. 15.

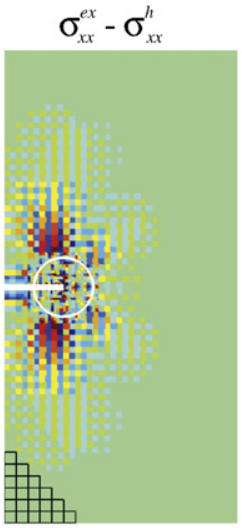

$[-32.19,105.81]$

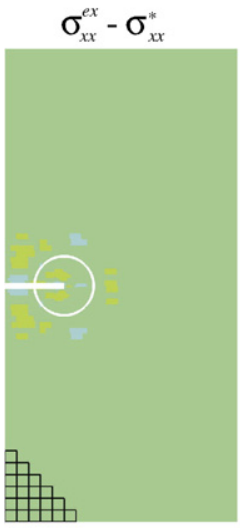

$[-6.31,3.90]$

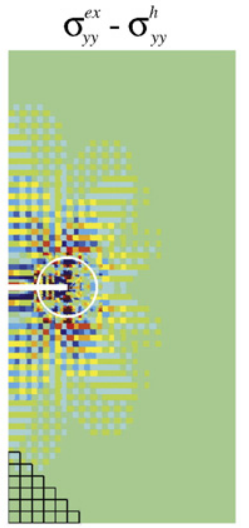

[-64.12, 156.78]

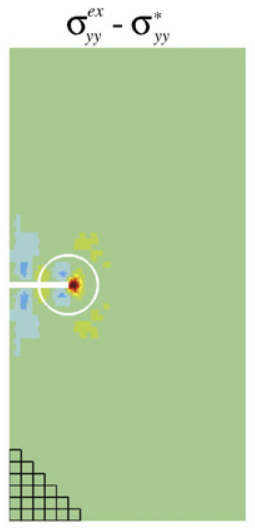

$[-2.87,10.49]$

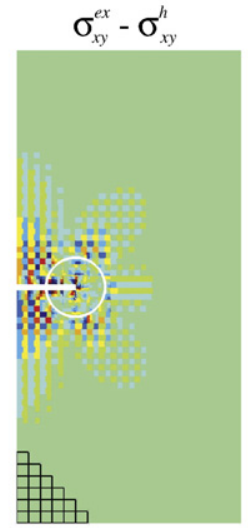

[-24.91, 24.91]

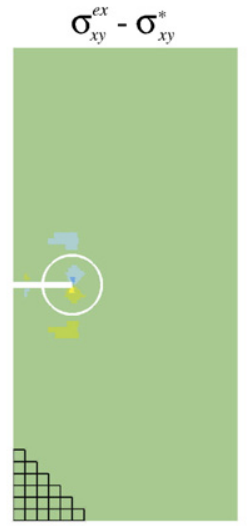

$[-2.86,2.86]$

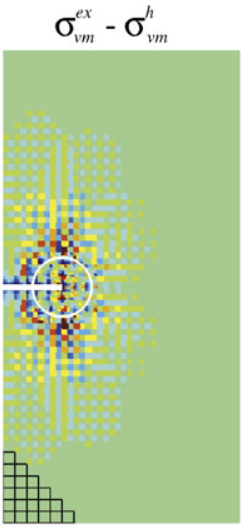

$[-32.19,105.81]$

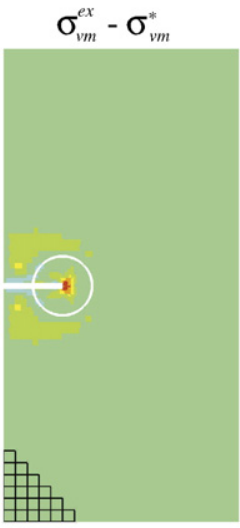

$[-6.29,7.12]$

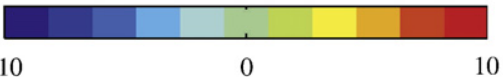

Fig. 11. Mode I, uniform mesh 2. Exact error in $\sigma_{x x}, \sigma_{y y}, \sigma_{x y}$ and $\sigma_{v m}$ obtained by XFEM and by means of the SPR-CX technique. Under each graph is indicated the range of the error in stresses. 


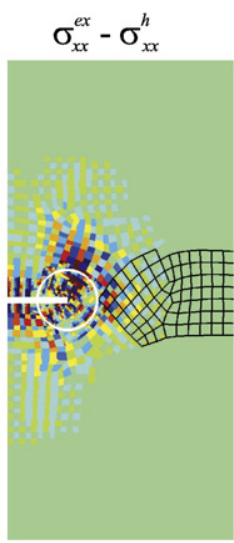

$[-152.43,190.22]$

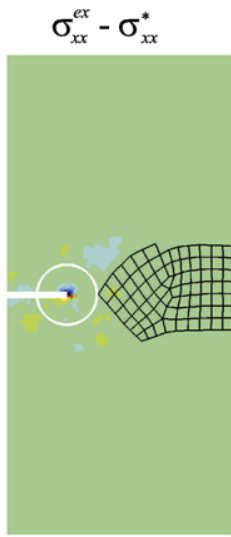

$[-21.84,15.71]$

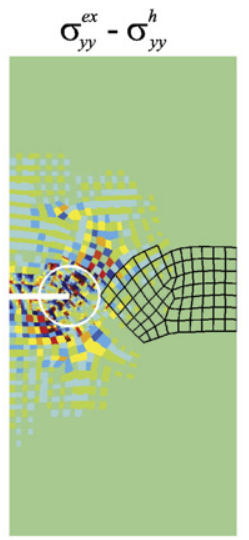

$[-114.42,127.96]$

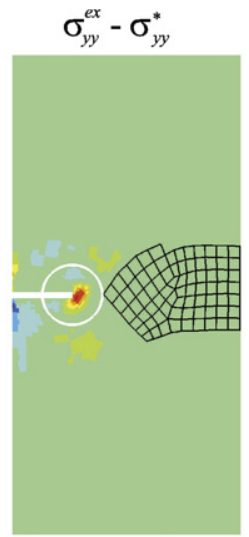

$[-7.86,9.69]$

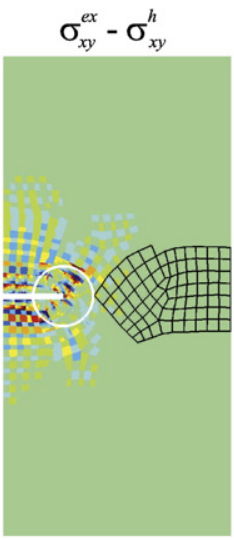

$[-70.85,160.05]$

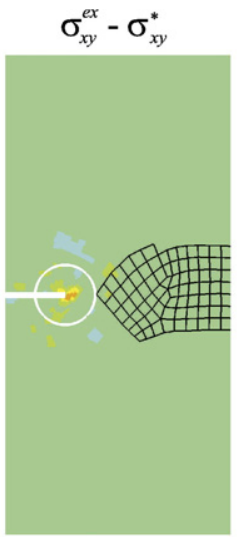

$[-1.48,5.27]$

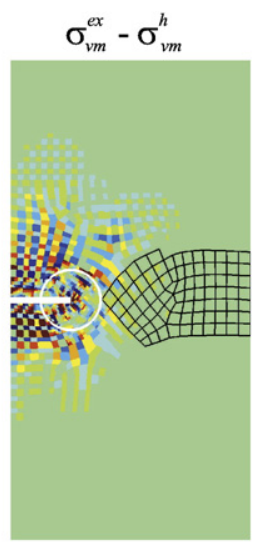

$[-133.83,304.25]$

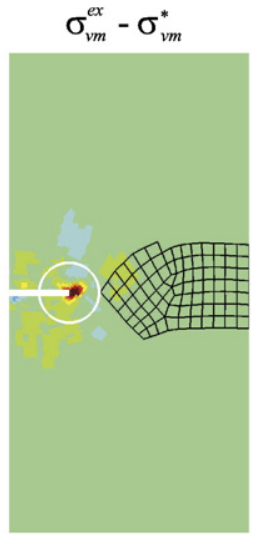

$[-5.73,21.83]$

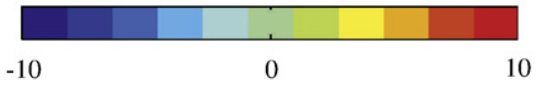

Fig. 12. Mixed mode, non-uniform mesh 2. Exact error in $\sigma_{x x}, \sigma_{y y}, \sigma_{x y}$ and $\sigma_{v m}$ obtained by XFEM and by means of the SPR-CX technique. Under each graph is indicated the range of the error in stresses.

5.4. Accuracy of the proposed upper error bound considering the exact error in the displacements

Fig. 15 shows the evolution of the error estimator $E_{S P R-C X}$ and for the bound $E_{U B e x}$ defined as in Eq. (27) and using the exact value of the error in the displacements field e, for Mode I.

The results displayed in Fig. 14 show effectivities of $E_{U B O}$ in the range $(1,1.80)$ whereas the results displayed in Fig. 15 show much more accurate results for $E_{U B e x}$, with effectivities in the range $(1,1.04)$, of the same order of magnitude than the results obtained for the error estimator $E_{S P R-C X}$.

The lack of accuracy of the results obtained through the use of Eq. (17) is mainly due to the use of the excessively conservative C S inequality in the evaluation of this expression, which strongly penalizes the evaluation of the upper bound.

\subsection{Effect of the correction terms due to lacks of equilibrium}

This section is used to analyze the effect of the integrals used to account for the equilibrium defects of $\boldsymbol{\sigma}^{*}$, into the domain and along the boundary, over the value of the upper error bound $E_{U B}$, see Eq. (27). Fig. 16 shows, in a logarithmic scale, the evolution of the absolute values of the correction terms when calculated both, using the exact values of the error in the displacements field $\mathbf{e}$ and its estimation $\mathbf{e}_{e s}$ evaluated as described in step 4 of Fig. 4, Section 4.2. The graph shows that the results obtained considering the estimate $\mathbf{e}_{e s}$ accurately reproduce those obtained with $\mathbf{e}$, demonstrating the validity of the technique used to obtain the estimate $\mathbf{e}_{e s}$. The graph also shows that the correction term due to lack of equilibrium along the boundary, associated with $\int_{\Gamma_{N}} \mathbf{e} \cdot \mathbf{r} \mathrm{d} \Gamma$ is about two orders of magnitude smaller than the correction term due to the lack of internal equilibrium, associated with $\int_{\Omega} \mathbf{e} \cdot \mathbf{s} \mathrm{d} \Omega$. Thus, one can reduce the computational cost of the proposed procedure assuming that $\int_{\Gamma_{N}} \mathbf{e} \cdot \mathbf{r} \mathrm{d} \Gamma \approx 0$, and rewriting Eq. (27) as

$\|\mathbf{e}\|^{2} \leq a\left(\boldsymbol{\sigma}_{e}^{*}, \boldsymbol{\sigma}_{e}^{*}\right)-2 \int_{\Omega} \mathbf{e} \cdot \mathbf{s} d \Omega=E_{U B}^{2}$.

Note that the value of $\int_{\Gamma_{N}} \mathbf{e} \cdot \mathbf{r} \mathrm{d} \Gamma$ can be neglected because the boundary equilibrium residual, $\mathbf{r}$, is very small as the recovery tech nique guarantees that $\boldsymbol{\sigma}^{*}$ exactly represents a 2 nd order Taylor's expansion of the stresses applied over the surface. This approximation is very accurate. In fact, this approximation ensures the exact satisfaction of the boundary equilibrium equation along the crack surfaces assuming that they are not in contact. On the other hand, the type of loads that cannot be represented by a second order polynomial, as those used in the numerical example (see Eq. (30)), will only appear in exceptional cases. In practical applications, the load distribution along the boundary, in general, can be exactly represented by a second order polynomial. Therefore, in these cases 

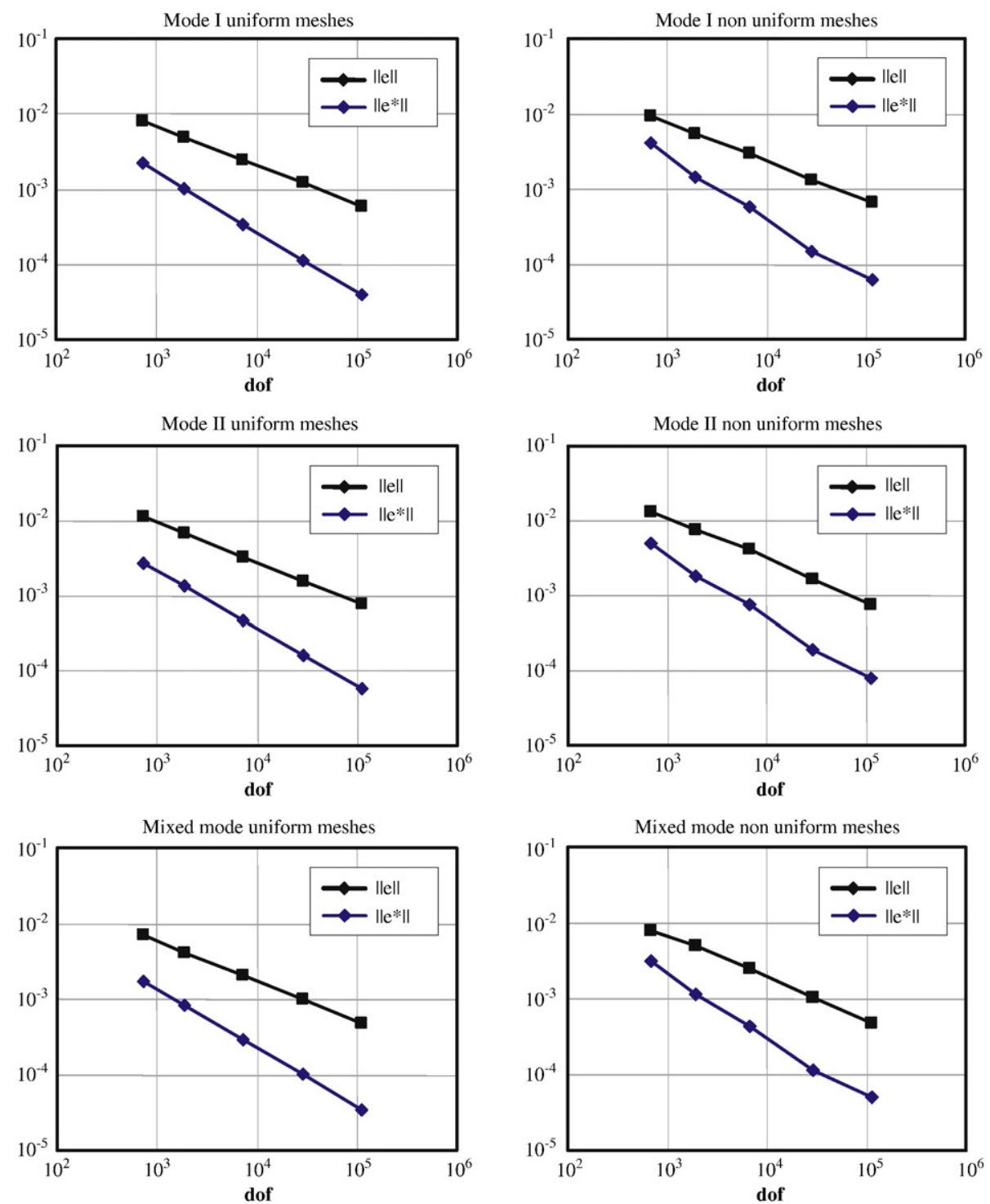

Fig. 13. Evolution of errors in energy norm.

there will be no residual associated with equilibrium defaults along the boundaries. If a less accurate recovery technique along the boundaries is used, the residual $\mathbf{r}$ will be higher. The evaluation of $\int_{\Gamma_{N}} \mathbf{e} \cdot \mathbf{r} \mathrm{d} \Gamma$ would then be required.

\subsection{Effectivity of the upper bound}

The accuracy of the upper bound of the discretization error has been studied in this section using the effectivity of the error estimator $\theta$, Mode I uniform meshes

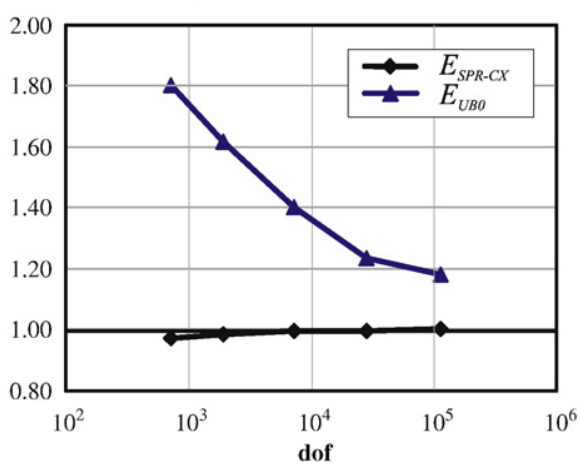

$\theta$, Mode I non uniform meshes

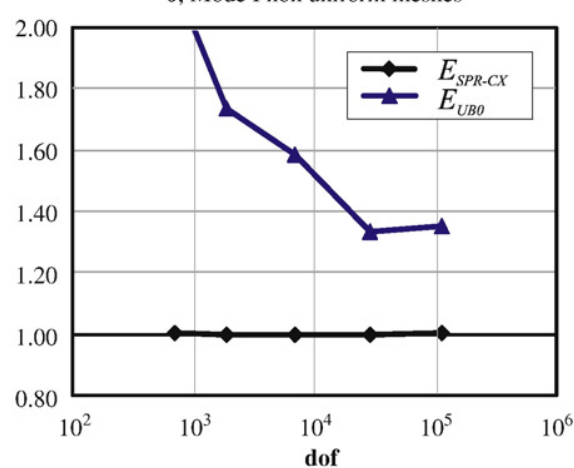

Fig. 14. Mode I. Evolution of the error estimator $E_{S P R} C X$ and the upper bound $E_{U B O}$. 

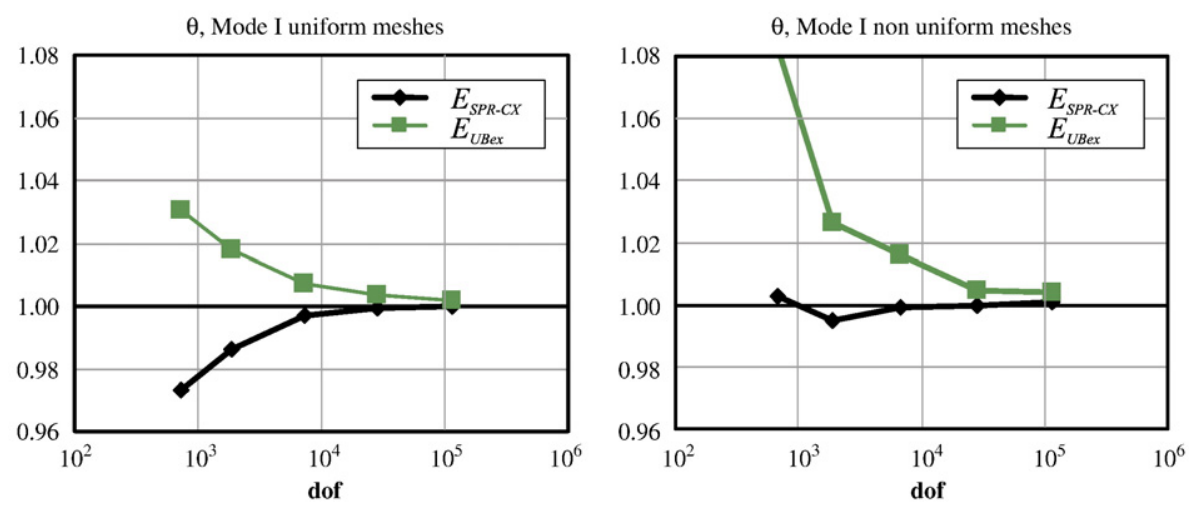

Fig. 15. Mode I. Evolution of $E_{S P R} C X$ and $E_{U B e x}$.

in the 3 problems under analysis (modes I and II and mixed mode) considering both, uniform and non uniform meshes. Four effectivity curves have been represented in each of the graphs of Fig. 17:

a) $E_{S P R-C X}$ curve: the effectivity of the error estimator,

b) $E_{\text {UBex }}$ curve: the effectivity of the upper error bound given by Eq. (27) evaluated using the exact value of the error of the displace ments field $\mathbf{e}$,

c) $E_{U B}$ curve: the effectivity of the upper error bound evaluated using Eq. (35) and the estimated error in the displacements field $\mathbf{e}_{e s}$ obtained from the last mesh of the overall refinement sequence, i.e., from the 5th mesh in all the problems analyzed,

d) $E_{U B S}$ curve: the effectivity of the upper error bound in Eq. (35) using the estimated error in the displacements field $\mathbf{e}_{e s}$ consider ing the last mesh available at each refinement step.

Remark 6. It is clear that using the last mesh of a refinement se quence to project the estimated error in the first mesh will provide good results. However, what it is interesting is to estimate the error in the best solution available at each refinement step. To evaluate $E_{U B s}$ we would need at least 3 meshes in order to obtain the convergence rate from the first 2 meshes and then to extrapolate the values of the correcting terms for the last mesh.

The following conclusions can be extracted from Fig. 17:

- The $E_{S P R-C X}$ curves show the high precision of the recovery technique used to evaluate $\boldsymbol{\sigma}^{*}$ both for uniform and non uniform meshes.

- Although $\boldsymbol{\sigma}^{*}$ is very accurate, the error estimator $E_{S P R-C X}^{2}=\overline{\mathrm{a}}\left(\boldsymbol{\sigma}_{e}^{*}, \boldsymbol{\sigma}_{e}^{*}\right)$ cannot be considered, by itself, as an upper error bound. While the effectivity of the error estimator is very close to 1 , in many cases is smaller than 1.

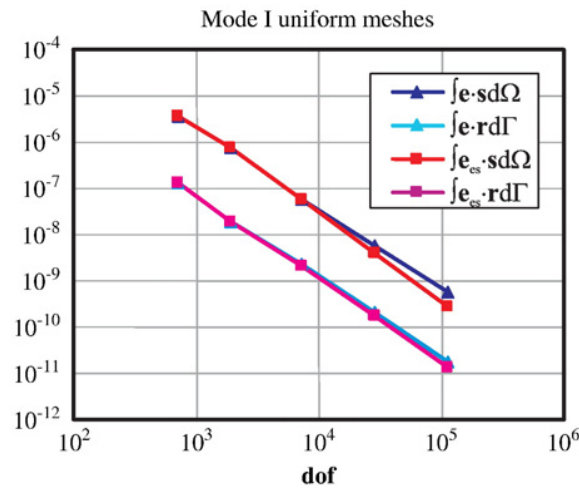

- The $E_{U B e x}$ curves numerically show that, for the different problems analyzed, Eq. (35) always provides an upper bound of the discretiza tion error in energy norm.

- The $E_{U B}$ curves, obtained using the estimation of the error in the displacements field described in Section 4.2, accurately reproduce the $E_{U B e x}$ curves evaluated with the exact values of the error. These results validate the use of the displacement error estimation tech nique, although the accuracy slightly diminishes in the last mesh of the sequence due to the Richardson extrapolation technique used in this mesh (see Table 1 ).

- The $E_{U B S}$ curves verify the accuracy of the proposed technique when the solution for a highly refined mesh used to project the displace ment error is not available. The effectivity in such cases is evaluated at each refinement step, obtaining values close to the effectivity of $E_{\text {UBex. }}$. Note that only in one case the computed version of the theoretical bound given by Eq. (27) provides effectivity values below unity $(\theta=0.996)$.

\section{Conclusions}

This paper presents a theoretical upper bounded estimator for the error in energy norm and a strategy to evaluate its computable version for LEFM problems solved using XFEM. The technique here proposed is an enhancement of a previous technique developed for FEM [31] and an adaptation to XFEM approximations.

The proposed procedure consists of two phases: a) evaluation of a nearly statically admissible recovered stress field that provides a very accurate estimation of the error in energy norm; and b) correction of the error estimation to account for the equilibrium defects of the nearly statically admissible field, which guarantees that the error obtained is an upper bound of the exact error.

The recovery technique providing the nearly statically admissible stress field is based on the SPR technique and the use of a splitting

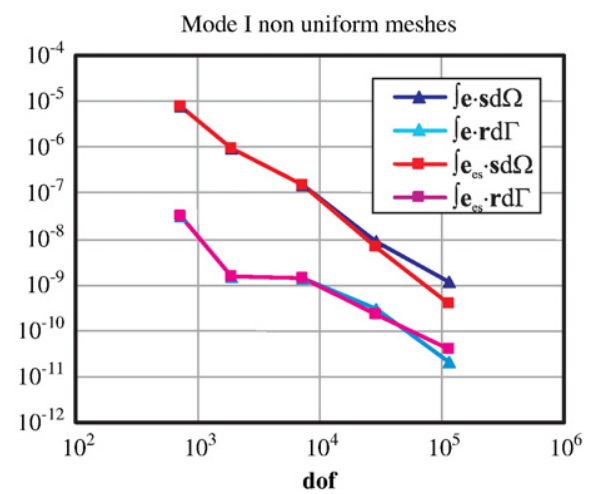

Fig. 16. Mode I. Evolution of the absolute value of the correction terms. 
$\theta$, Mode I uniform meshes

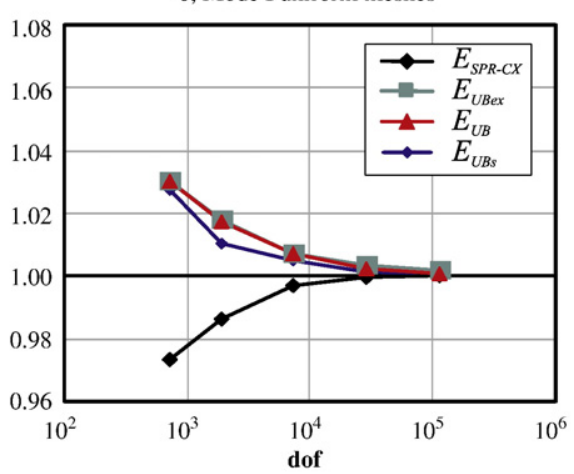

$\theta$, Mode II uniform meshes

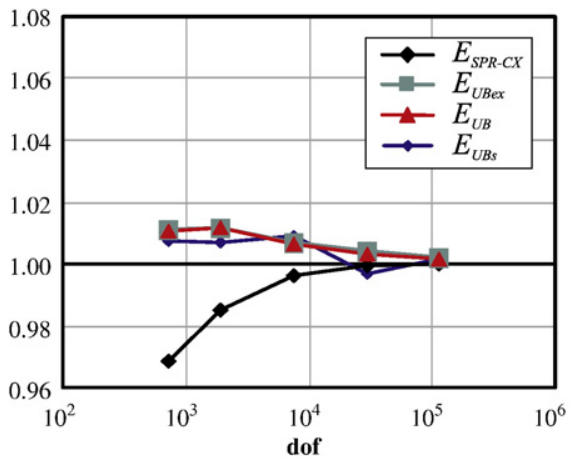

$\theta$, Mixed Mode uniform meshes

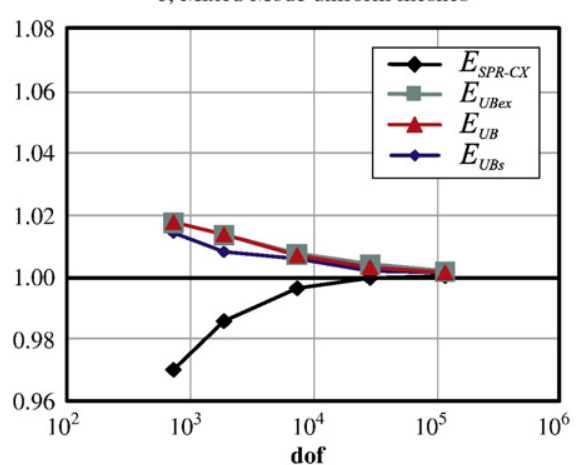

$\theta$, Mode I non uniform meshes

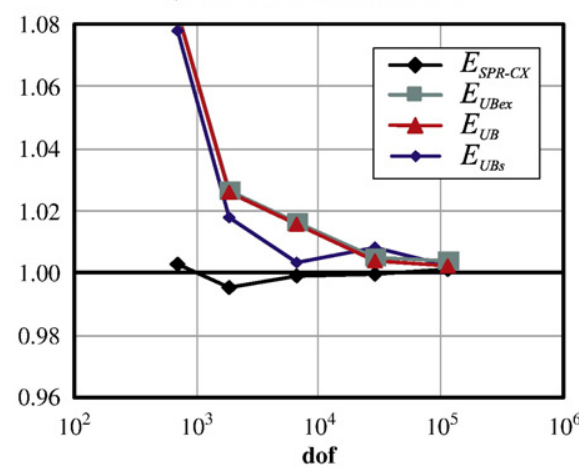

$\theta$, Mode II non uniform meshes

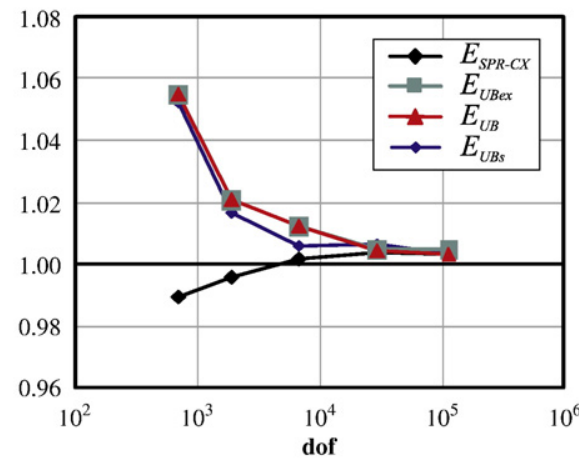

$\theta$, Mixed Mode non uniform meshes

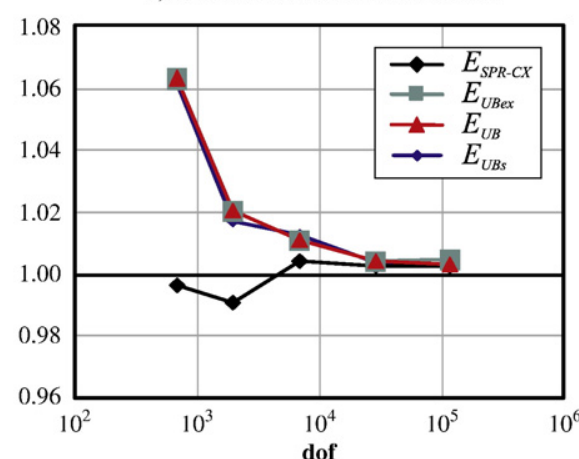

Fig. 17. Modes I, II and mixed, sequences of uniform and non-uniform meshes. Evolution of the effectivities of the error estimator $E_{S P R} C X$ and the upper bounds $E_{U B e x}, E_{U B}$ and $E_{U B s}$.

technique that decomposes the stress field into a singular part and a smooth part. The proposed procedure uses a recovery technique which ensures that, at each patch, $\boldsymbol{\sigma}_{i}^{*}$ is statically admissible, matching body loads and boundary tractions. Finally, a PUM based technique is used to obtain a continuous recovered field $\boldsymbol{\sigma}^{*}$, which, on the other hand, introduces lacks of equilibrium in the recovered solution.

Table 1

Effectivities of the error estimator $E_{S P R} C X$ and the upper bounds $E_{U B e x}$ and $E_{U B}$ for the last mesh in the uniform and non-uniform sequences.

\begin{tabular}{lllll}
\hline & dof & $\theta_{S P R C X}$ & $\theta_{\text {UBex }}$ & $\theta_{U B}$ \\
\hline Uniform meshes & & & & \\
Mode I & 113,477 & 1.00017 & 1.00176 & 1.00091 \\
Mode II & 113,477 & 1.00033 & 1.00239 & 1.00167 \\
Mixed mode & 113,477 & 1.00027 & 1.00215 & 1.00137 \\
& & & & \\
Non-uniform meshes & & & & \\
Mode I & 113,991 & 1.00127 & 1.00415 & 1.00214 \\
Mode II & 113,991 & 1.00334 & 1.00506 & 1.00335 \\
Mixed mode & 113,991 & 1.00278 & 1.00476 & 1.00310 \\
\hline
\end{tabular}

A new technique for the evaluation of the correction terms associat ed with the lack of equilibrium has also been presented. The technique requires the problem to be solved using a sequence of refined meshes. The numerical experiments show the high accuracy of the proposed technique. The need for a mesh sequence and the lack of accuracy in the last mesh suggest developing new procedures for the estimation of the error in the displacement field, without using any mesh sequence. This is left for future research.

The numerical results show that the estimation of the discretization $E_{S P R-C X}$ is very accurate, with effectivities within the range (0.95-1.01). Despite of the fact that $E_{S P R-C X}$ is not an upper bound of the exact error, the numerical results indicate that this value could be considered as a low cost computed version of the theoretical upper bound where the effect of all the correction terms due to equilibrium defects has been neglected.

\section{Acknowledgements}

This work has been carried out within the framework of the re search projects DPI2007 66773 C02 01, DPI2007 66995 C03 02 and DPI2007 62395 of the Ministerio de Educación y Ciencia (Spain). 
The financial support of the Generalitat Valenciana and the Universidad Politécnica de Valencia is also acknowledged.

\section{References}

[1] N. Moës, J. Dolbow, T. Belytschko, A finite element method for crack growth without remeshing, Int. J. Numer. Meth. Eng. 46 (1999) 131-150.

[2] J.M. Melenk, I. Babuška, The partition of unity finite element method: basic theory and applications, Comput. Meth. Appl. Mech. Eng. 139 (1996) 289-314.

[3] B.L. Karihaloo, Q.Z. Xiao, Modelling of stationary and growing cracks in FE framework without remeshing: a state-of-the-art review, Comput. Struct. 81 (2003) 119-129.

[4] N. Moës, A. Gravouil, T. Belytschko, Non-planar 3D crack growth by the extended finite element and level sets - part I: mechanical model, Int. J. Numer. Meth. Eng. 53 (2002) 2549-2568.

[5] A. Gravouil, T. Belytschko, N. Moës, Non-planar 3D crack growth by the extended finite element and level sets - part II: level set update, Int. J. Numer. Meth. Eng. 53 (2002) 2569-2586.

[6] D.L. Chopp, N. Sukumar, Fatigue crack propagation of multiple coplanar cracks with the coupled extended finite element/fast marching method, Int. J. Eng. Sci. 41 (2003) 845-869.

[7] P. Laborde, J. Pommier, Y. Renard, M. Salaün, High-order extended finite element method for cracked domains, Int. J. Numer. Meth. Eng. 64 (2005) 354-381.

[8] E. Béchet, H. Minnebo, N. Moës, B. Burgardt, Improved implementation and robustness study of the X-FEM method for stress analysis around cracks, Int. J. Numer. Meth. Eng. 64 (2005) 1033-1056.

[9] T. Strouboulis, K. Copps, I. Babuška, The generalized finite element method, Comput. Meth. Appl. Mech. Eng. 190 (2001) 4081-4193.

[10] Q.Z. Xiao, B.L. Karihaloo, Improving the accuracy of XFEM crack tip fields using higher order quadrature and statically admissible stress recovery, Int. J. Numer. Meth. Eng. 66 (2006) 1378-1410.

[11] J.E. Tarancón, A. Vercher, E. Giner, F.J. Fuenmayor, Enhanced blending elements for XFEM applied to linear elastic fracture mechanics, Int. J. Numer. Meth. Eng. 77 (2009) $126-148$

[12] M. Ainsworth, J.T. Oden, A Posteriori Error Estimation in Finite Element Analysis, John Wiley \& Sons, Chichester, 2000

[13] W. Bangerth, R. Rannacher, Adaptive Finite Element Methods for Differential Equations, ETH, Zürich, Birkhäuser, Basel, 2003.

[14] O.C. Zienkiewicz, J.Z. Zhu, A simple error estimation and adaptive procedure for practical engineering analysis, Int. J. Numer. Meth. Eng. 24 (1987) 337-357.

[15] O.C. Zienkiewicz, J.Z. Zhu, The superconvergent patch recovery and a posteriori error estimates. Part I: the recovery technique, Int. J. Numer. Meth. Eng. 33 (1992) $1331-1364$

[16] O.C. Zienkiewicz, J.Z. Zhu, The superconvergent patch recovery and a posteriori error estimates. Part II: error estimates and adaptivity, Int. J. Numer. Meth. Eng. 33 (1992) 1365-1382.

[17] I. Babuška, T. Strouboulis, C.S. Upadhyay, A model study of the quality of $a$ posteriori error estimators for linear elliptic problems. Error estimation in the interior of patchwise uniform grids of triangles, Comput. Meth. Appl. Mech. Eng. 114 (1994) 307-378.

[18] I. Babuška, T. Strouboulis, C.S. Upadhyay, J. Gangaraj, K. Copps, Validation of $a$ posteriori error estimators by numerical approach, Int. J. Numer. Meth. Eng. 37 (1994) 1073-1123.

[19] N.E. Wiberg, F. Abdulwahab, S. Ziukas, Enhanced superconvergent patch recovery incorporating equilibrium and boundary conditions, Int. J. Numer. Meth. Eng. 37 (1994) 3417-3440.

[20] A.C.A. Ramsay, E.A.W. Maunder, Effective error estimation from continuous, boundary admissible estimated stress fields, Comput. Struct. 61 (1996) 331-343.

[21] T. Blacker, T. Belytschko, Superconvergent patch recovery with equilibrium and conjoint interpolant enhancements, Int. J. Numer. Meth. Eng. 37 (1994) 517-536.

[22] Q.Z. Xiao, B.L. Karihaloo, Statically admissible stress recovery using the moving least squares technique, in: B.H.V. Topping, C.A. Mota Soares (Eds.), Progress in
Computational Structures Technology, Saxe-Coburg Publications, Stirling, Scotland, 2004, pp. 111-138.

[23] J.J. Ródenas, M. Tur, F.J. Fuenmayor, A. Vercher, Improvement of the superconvergent patch recovery technique by the use of constraint equations: the SPRC technique, Int. J. Numer. Meth. Eng. 70 (2006) 705-727.

[24] J.J. Ródenas, E. Giner, J.E. Tarancón, O.A. González-Estrada, A recovery error estimator for singular problems using singular + smooth field splitting, in: B.H.V Topping, G. Montero, R. Montenegro (Eds.), Proceedings of Fifth International Conference on Engineering Computational Technology, Fifth International Conference on Engineering Computational Technology, Civil-Comp Press, Stirling, Scotland, 2006

[25] C. Shih, R. Asaro, Elastic-plastic analysis of cracks on bimaterial interfaces: part I small scale yielding, J. Appl. Mech. 8 (1988) 537-545.

[26] J. Yau, S. Wang, H. Corten, A mixed-mode crack analysis of isotropic solids using conservation laws of elasticity, J. Appl. Mech. 47 (1980) 335-341.

[27] P. Díez, J.J. Egozcue, A. Huerta, A posteriori error estimation for standard finite element analysis, Comput. Meth. Appl. Mech. Eng. 163 (1998) 141-157.

[28] P. Díez, N. Parés, A. Huerta, Recovering lower bounds of the error by postprocessing implicit residual a posteriori error estimates, Int. J. Numer. Meth. Eng. 56 (2003) 1465-1488.

[29] J.P.M. de Almeida, O.J.B.A. Pereira, Upper bounds of the error in local quantities using equilibrated and compatible finite element solutions for linear elastic problems, Comput. Methods Appl. Mech. Eng. 195 (2006) 279-296.

[30] O.JB.A. Pereira, J.P.M. de Almeida, E.A.W. Maunder, Adaptive methods for hybrid equilibrium finite element models, Comput. Meth. Appl. Mech. Eng. 176 (1999) 19-39.

[31] P. Díez, J.J. Ródenas, O.C. Zienkiewicz, Equilibrated patch recovery error estimates: simple and accurate upper bounds of the error, Int. J. Numer. Meth. Eng. 69 (2007) 2075-2098.

[32] T. Strouboulis, L. Zhang, D. Wang, I. Babuška, A posteriori error estimation for generalized finite element methods, Comput. Meth. Appl. Mech. Eng. 195 (2006) 852-879.

[33] S. Bordas, M. Duflot, P. Le, A simple error estimator for extended finite elements, Commun. Numer. Meth. Eng. 24 (2008) 961-971.

[34] S. Bordas, M. Duflot, Derivative recovery and a posteriori error estimate for extended finite elements, Comput. Meth. Appl. Mech. Eng. 196 (2007) 3381-3399.

[35] M. Duflot, S. Bordas, A posteriori error estimation for extended finite element by an extended global recovery, Int. J. Numer. Meth. Eng. 76 (2008) 1123-1138.

[36] J. Panetier, P. Ladevèze, L. Chamoin, Strict and effective bounds in goal-oriented error estimation applied to fracture mechanics problems solved with XFEM, Int. J. Numer. Meth. Eng. 81 (2010) 671-700.

[37] J.J. Ródenas, O.A. González-Estrada, J.E. Tarancón, F.J. Fuenmayor, A recovery type error estimator for the extended finite element method based on a singular + smooth stress field splitting, Int. J. Numer. Meth. Eng. 76 (2008) 545-571.

[38] M.F. Kanninen, C.H. Popelar, Advanced Fracture Mechanics, Oxford University Press, New York, 1985

[39] T. Belytschko, T. Black, Elastic crack growth in finite elements with minimal remeshing, Int. J. Numer. Meth. Eng. 45 (1999) 601-620.

[40] M. Stolarska, D.L. Chopp, N. Moës, T. Belytschko, Modelling crack growth by leve sets and the extended finite element method, Int. J. Numer. Meth. Eng. 51 (2001) 943-960.

[41] L. Banks-Sills, Application of the finite element method to linear elastic fracture mechanics, Appl. Mech. Rev. 44 (1991) 447-461.

[42] F.Z. Li, C.F. Shih, A. Needleman, A comparison of methods for calculating energy release rates, Eng. Fract. Mech. 21 (1985) 405-421

[43] G. Calderón, P. Díez, Análisis de diferentes estimadores de error de postproces para adaptatividad orientada al resultado, Métodos Numéricos Para Cálculo y Diseño En Ingeniería 22 (2006) 193.

[44] E. Giner, F.J. Fuenmayor, L. Baeza, J.E. Tarancón, Error estimation for the finite element evaluation of $G_{I}$ and $G_{I I}$ in mixed-mode linear elastic fracture mechanics, Finite Elem. Anal. Des. 41 (2005) 1079-1104. 